\title{
ER-associated CTRP1 regulates mitochondrial fission via interaction with DRP1
}

Seong Keun Sonn ${ }^{1}$, Seungwoon Seo ${ }^{1}$, Jaemoon Yang ${ }^{2}$, Ki Sook $\mathrm{Oh}^{3}$, Hsiuchen Chen ${ }^{4}$, David C. Chan ${ }^{4}$, Kunsoo Rhee (DD ${ }^{5}$, Kyung S. Lee ${ }^{6}$, Young Yang ${ }^{3 凶}$ and Goo Taeg Oh (D) ${ }^{1 凶}$

(c) The Author(s) 2021

C1q/TNF-related protein 1 (CTRP1) is a CTRP family member that has collagenous and globular C1q-like domains. The secreted form of CTRP1 is known to be associated with cardiovascular and metabolic diseases, but its cellular roles have not yet been elucidated. Here, we showed that cytosolic CTRP1 localizes to the endoplasmic reticulum (ER) membrane and that knockout or depletion of CTRP1 leads to mitochondrial fission defects, as demonstrated by mitochondrial elongation. Mitochondrial fission events are known to occur through an interaction between mitochondria and the ER, but we do not know whether the ER and/or its associated proteins participate directly in the entire mitochondrial fission event. Interestingly, we herein showed that ablation of CTRP1 suppresses the recruitment of DRP1 to mitochondria and provided evidence suggesting that the ER-mitochondrion interaction is required for the proper regulation of mitochondrial morphology. We further report that CTRP1 inactivation-induced mitochondrial fission defects induce apoptotic resistance and neuronal degeneration, which are also associated with ablation of DRP1. These results demonstrate for the first time that cytosolic CTRP1 is an ER transmembrane protein that acts as a key regulator of mitochondrial fission, providing new insight into the etiology of metabolic and neurodegenerative disorders.

Experimental \& Molecular Medicine (2021) 53:1769-1780; https://doi.org/10.1038/s12276-021-00701-z

\section{INTRODUCTION}

CTRP1 belongs to the C1q/TNF family, the members of which have four structurally distinct domains: a signal peptide at the $\mathrm{N}$ terminus, a short variable region, a collagenous domain and a C-terminal globular domain. CTRP1 levels were reportedly increased in the plasma or sera of patients with type II diabetes and ob/ob mice ${ }^{1-3}$. Circulating CTRP1 levels were also found to be increased in the sera of patients with coronary artery disease ${ }^{4-8}$ and heart disease ${ }^{9}$, suggesting that this protein has proatherogenic and cardiac effects. CTRP1 has been shown to regulate vascular function ${ }^{10-12}$ and protect against acute ischemic injury in the heart ${ }^{13}$. CTRP1 levels were reportedly increased in the sera of hypertensive patients, and CTRP1 has been shown to stimulate aldosterone production and plays a role in blood pressure homeostasis ${ }^{14,15}$. These findings suggest that the secreted form of CTRP1 plays important roles in regulating metabolic and cardiovascular functions. Regarding other family members, CTRP3 appears to promote energy production by inducing mitochondrial ROS, upregulating the expression of PGC-1a in vascular smooth muscle cells and inducing mitochondrial biogenesis in cardiomyocytes ${ }^{16,17}$. CTRP5 was increased in mitochondrial DNAdepleted myocytes and was shown to activate AMP-activated protein kinase ${ }^{18}$. Interestingly, Innamorati and colleagues suggested that GIF (G protein-interacting protein, which is another name for the above-described (TRP1) has a putative transmembrane domain and is highly expressed in the mitochondria-rich heart and other muscles ${ }^{19}$. This result suggested that cytosolic CTRP1 may modulate mitochondrial functions to regulate metabolic and cardiovascular functions. However, the cellular and molecular functions of cytosolic CTRP1 in mitochondrial dynamics have not yet been determined.

The dysregulation of mitochondrial fusion/fission dynamics has been associated with physiological effects, such as aging, as well as pathologies such as cardiovascular disease, metabolic disease and neurodegenerative diseases (e.g., Parkinson's, Huntington's and Alzheimer's diseases) ${ }^{20-22}$. In the fission process, DRP1 oligomerizes and drives mitochondrial fission at mitochondrial fission sites ${ }^{23,24}$. MFF, MiD49 (also known as MIEF1) and MiD51 (also known as MIEF2) regulate mitochondrial localization and the GTPase-mediated activation of DRP1 ${ }^{25,26}$. Although numerous DRP1 puncta localize to mitochondrial constriction sites, mitochondrial fission occurs infrequently, suggesting that additional events may be required to complete the fission process. The ER and mitochondria form extensive contacts with one another and show close dynamic interactions. ER tubules wrap around mitochondrial constrictions and fission sites ${ }^{27}$. Two proteins, ERbound inverted formin ( $\mathrm{FH} 2$ and $\mathrm{WH} 2$ domain-containing; IFN2) and mitochondria-bound spire $1 \mathrm{C}$, contribute to mitochondrial constriction through actin polymerization and thereby support DRP1 oligomerization ${ }^{28,29}$. However, no previous study has

\footnotetext{
${ }^{1}$ Department of Life Science, Heart-Immune-Brain Network Research Center, Ewha Woman's University, Seoul 03760, Republic of Korea. ${ }^{2}$ Department of Radiology, Yonsei University, Seoul 120-752, Republic of Korea. ${ }^{3}$ Department of Life Science, Research Center for Women's Disease, Sookmyung Women's University, Seoul, Republic of Korea. ${ }^{4}$ Division of Biology, California Institute of Technology, Pasadena, CA 91125, USA. ${ }^{5}$ Department of Biological Sciences, Seoul National University, Seoul, Republic of Korea.

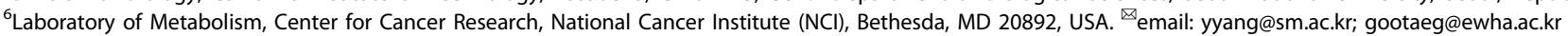


identified an ER protein that is directly associated with DRP1 assembly and affects mitochondrial fission. The literature indicates that DRP1 deficiency induces apoptotic resistance by influencing Bax oligomerization at the mitochondrial fission site ${ }^{30}$. Moreover, the DRP1 adaptors MiD49 and MiD51 have been shown to be essential for crista remodeling during apoptosis, and ablation of MFF, MiD49 and MiD51 was shown to affect resistance to cell death ${ }^{25,26}$. A central process in apoptosis is the release of cytochrome $\mathrm{c}$ from mitochondrial cristae into the cytoplasm. This release, which occurs through the mitochondrial outer membrane pores generated by BAX and BAK, initiates caspase cascade activation, leading to apoptosis ${ }^{31,32}$.

In this study, we examined the detailed role of cytosolic CTRP1. Our novel results show that CTRP1 localizes to the ER membrane and regulates mitochondrial fission through an interaction with the mitochondrial fission protein DRP1. Based on this finding, we further assessed whether CTRP1 plays a critical role in mitochondrial fission by examining the functional interaction between CTRP1 and DRP1 at ER-mitochondria contact sites (EMCSs).

\section{MATERIALS AND METHODS}

\section{Mice}

$C \operatorname{trp} 1^{\mathrm{fl} / \mathrm{fl}}$ mice were generated through a standard gene-targeting strategy using embryonic stem cells (clone E14). The targeting vector for the conditional allele was from the Knockout Mouse Project (KOMP) and contained a neomycin-resistance marker flanked by FRT and loxP sites that were inserted next to exons 2 and 4 of Ctrp1. Mice were backcrossed more than seven times onto the C57BL/6J background (Jackson Laboratory) and were crossed to a Flp recombinase-expressing transgenic mouse strain (Jackson Laboratory). FRT recombination was confirmed by PCR. Pcp2-Cre; Ctrp $1^{\mathrm{flfl}}$ and Alb-Cre;Ctrp $1^{\mathrm{f} / \mathrm{fl}}$ mice were generated by crossing Ctrp $1^{1 / f 1}$ mice with Pcp2-Cre or Alb-Cre transgenic strains of mice (Jackson Laboratory). The success of the crosses was confirmed by PCR. The PCR primers used were as follows: loxP site primer: LR- $5^{\prime}$-ACT GAT GGC GAG CTC AGA CC-3'; genomic primer: DF-5'-GCA CAC CTG TAT ACC AGA CA-3', genomic primer: DF2-5'-GCA CAC CTG TAT ACC AGA CA-3'; genomic primer: DR-5'-GAG GGA GAG AAG AAA GCC TA-3'. All animal care and experimental procedures were performed in compliance with protocols approved by the Institutional Animal Care and Use Committee of Ewha Woman's University.

\section{Plasmids and RNAi oligonucleotides}

GFP-CTRP1, BFP-CTRP1, CTRP1-Flag, Flag-tagged CTRP1 deletion mutant (1-98, 1-138, 99-281, 139-281, 20-281 and 31-281), and Myc- and Histagged CTRP1 deletion mutant (1-98, 1-138, 99-281, 139-281, 20-281 and 31-281) proteins were generated by PCR amplification of full-length CTRP1 (isoform 1; NCBI accession number NM_030968.3) and cloning of the product into the EcoRI-BamHI sites or BamHI-EcoRI sites of the pEGFP-N3 (Clontech), pEGFP-C1 (Clontech), pCMV-Tag 4A (Stratagene) or pcDNA.3.1/ Myc-His(-) A (Invitrogen) plasmids. Point mutations in CTRP1 (for expression of $\mathrm{CTRP}_{1}{ }^{\mathrm{C1} \mathrm{R}}$ and $\mathrm{CTRP} 1^{\mathrm{C} 13 \mathrm{~A}}$ ) were generated with a Phusion Site-directed Mutagenesis Kit according to the manufacturer's protocol (Thermo Fisher Scientific, Inc.). The construct encoding prohibitin-Myc-His was generated by PCR amplification of full-length prohibitin (isoform 1; NCBI accession number NM_001281496.1) and cloning the product into the EcoRI-BamHI sites of the pcDNA.3.1/Myc-His(-) A plasmid (Invitrogen). The construct encoding DRP1-GFP was generated by PCR amplification of full-length Drp1 (isoform 3; NCBI accession number NM_005690.4) and cloning the product into the EcoRI-BamHI sites of the pEGFP-C1 plasmid (Clontech). The construct encoding MFF-GFP was generated by PCR amplification of full-length MFF (isoform a; NCBI accession number NM 001277061.1) and cloning the product into the Xhol-BamHI sites of the pEGFP-C1 plasmid (Clontech). The construct encoding FIS1-GFP was generated by PCR amplification of full-length Fis 1 (NCBI accession number NM_016068.2) and cloning the product into the EcoRI-BamHI sites of the pEGFP-C1 plasmid (Clontech). The construct encoding MiD49-GFP was generated by PCR amplification of full-length MiD49 (isoform 1; NCBI accession number NM_139162.3) and cloning the product into the EcoRI-BamHI sites of the pEGFP-C1 plasmid (Clontech). The construct encoding MiD51-GFP was generated by PCR amplification of full-length MiD51 (isoform 1; NCBI accession number 2.NM_019008.5) and cloning the product into the EcoRI-BamHI sites of the pEGFP-C1 plasmid (Clontech). The construct encoding mtPAGFP was provided by Addgene. The siRNAs were synthesized by GenePharma, and the sequences used were as follows: siCTRP1 against the target sequence $5^{\prime}$-CCGGCAAGTTCTAC TGCTAtt- ${ }^{\prime}$, siDRP1 against the target sequence 5'-AACGCAGAGCAGCGG AAAGAGtt- 3 ', siMFF against the target sequence $5^{\prime}$ - AACGCTGACCTGGA ACAAGGAtt-3', siMiD49 against the target sequence $5^{\prime}$-GCAGTATGAG CGTGACAAAAtt-3', siMiD51 against the target sequence $5^{\prime}$-GATTGAC GACATTGGCUATtt- $3^{\prime}$, sifis1 against the target sequence 5'-GTACAATGATGACATCCGTAAtt- 3 ', siBak against the target sequence $5^{\prime}$ AATGCCTATGAGTACTTCACCtt- ${ }^{\prime}$, and siBax against the target sequence $5^{\prime}$-TATGGAGCTGCAGAGGATGtt-3'. As a control, Silencer Negative Control siRNA (GenePharma) was used.

\section{Mammalian cell growth, transfection and drug treatment}

Primary MEFs were isolated from wild-type and $C t r p 1^{f / f}$ embryos. Primary MEFs, HeLa cells, 293T cells and U2OS cells were maintained in Dulbecco's modified Eagle's medium (DMEM; Invitrogen) supplemented with 10\% FBS (Invitrogen), $100 \mathrm{U} / \mathrm{ml}$ penicillin and $100 \mathrm{\mu g} / \mathrm{ml}$ streptomycin (Invitrogen). For inactivation of endogenous Ctrp1, Ctrp $1^{\mathrm{fl} / \mathrm{fl}}$ MEFs were infected with a Cre-expressing (Puro) lentivirus (Genetarget) at a dose of $1 \times 10^{7}$ plaqueforming units and cultured for $24 \mathrm{~h}$ in DMEM supplemented with $10 \% \mathrm{FBS}$ and $3 \mu \mathrm{g} / \mathrm{ml}$ puromycin (Sigma). Cells $\left(2 \times 10^{5}\right.$ cells/well) were seeded in a six-well plate for $18 \mathrm{~h}$ prior to transfection. Plasmid transfections were performed in Opti-MEM medium (Invitrogen) with $3 \mu \mathrm{l}$ of Lipofectamine 2000 (Invitrogen) for $4 \mathrm{~h}$. The cells were then seeded onto microscope dishes with cover-glass bottoms (SPL) at a concentration of $\sim 0.5 \times 10^{5}$ cells $/ \mathrm{ml}$. For RNAi transfections, cells were seeded onto six-well plates at $30-40 \%$ density (or an equivalent density). Then, $4 \mu$ of Lipofectamine 2000 (Invitrogen) and $40 \mathrm{nM}$ RNAi oligonucleotides were added to each well. Cells were retransfected $24 \mathrm{~h}$ later with $3 \mu \mathrm{l}$ of Lipofectamine 2000 and the appropriate plasmid DNA. For mitochondrial staining, cells were treated with $100 \mathrm{nM}$ MitoTracker Red CMXRos (Invitrogen) in DMEM for $20 \mathrm{~min}$ prior to fixation. For staurosporine (STS; Sigma), actinomycin D (Sigma) or etoposide (Sigma) treatment, cells were seeded on poly-L-lysinecoated coverslips $(0.1 \mathrm{mg} / \mathrm{ml}$, Sigma) and incubated with $1 \mu \mathrm{M}$ staurosporine for $4 \mathrm{~h}, 10 \mu \mathrm{M}$ actinomycin $\mathrm{D}$ for $8 \mathrm{~h}$ or $20 \mu \mathrm{M}$ etoposide for $16 \mathrm{~h}$ in serum-containing medium. Treatment with DMSO was used as the negative control.

\section{Electron microscopy}

For preparation of samples for cellular transmission electron microscopy (TEM), MEFs $\left(5.0 \times 10^{5}\right.$ cells/well) were seeded onto six-well plates for $24 \mathrm{~h}$. The rabbit polyclonal antibody to CTRP1 was reacted with a 1:3 dilution of 5-nm gold-conjugated goat anti-rabbit lgG (Invitrogen) or 10-nm goldconjugated goat anti-rabbit lgG (Sigma) in DMEM for $1 \mathrm{~h}$ at $37^{\circ} \mathrm{C}$. The cells were then rinsed with PBS ( $\mathrm{pH} 7.4,1 \mathrm{mM})$, and gold-conjugated polyclonal anti-CTRP1 was added to DMEM supplemented with $10 \%$ FBS. After $6 \mathrm{~h}$ of incubation at $37^{\circ} \mathrm{C}$, the cells were washed three times with PBS, centrifuged and then washed three times with blocking buffer $(0.03 \%$ bovine serum albumin and $0.01 \% \mathrm{NaN}_{3}$ in PBS). The samples were then fixed with $2 \%$ glutaraldehyde paraformaldehyde in $0.1 \mathrm{M} \mathrm{PBS}(\mathrm{pH} 7.4)$ for $2 \mathrm{~h}$ and washed three times for $30 \mathrm{~min}$ in $0.1 \mathrm{M}$ PBS (pH 7.4, $1 \mathrm{mM}$ ). The cells were then postfixed with $1 \% \mathrm{OsO}_{4}$ (osmium tetroxide) dissolved in $0.1 \mathrm{M} \mathrm{PBS}(\mathrm{pH} 7.4)$ for $2 \mathrm{~h}$, dehydrated in an ascending gradual series $(50 \%$, $60 \%, 70 \%, 80 \%, 90 \%, 95 \%$ and $100 \%$ ) of ethanol and infiltrated with propylene oxide. Specimens were embedded using a Poly/Bed 812 kit (Polysciences, USA). After the samples were embedded in pure, fresh resin at $60^{\circ} \mathrm{C}$ in an electron microscope oven (TD-700, Dosaka, Japan) for $24 \mathrm{~h}$, 300-nm-thick sections were initially cut and stained with toluidine blue for observation under a light microscope (Olympus BX40, Japan). Then, 80$\mathrm{nm}$-thick sections were double-stained with $7 \%$ uranyl acetate and lead citrate for contrast staining $(20 \mathrm{~min})$. The sections were then cut using a Leica Ultracut UCT Ultramicrotome (Leica Microsystems, Austria). All samples were observed using TEM (JEM-1011, JEOL, Japan) at an acceleration voltage of $80 \mathrm{kV}$.

\section{Subcellular fractionation}

As previously described ${ }^{33,34}$, MEFs or U2OS cells were washed with PBS $\left(\mathrm{Ca}^{2+}\right.$ - and $\mathrm{Mg}^{2+}$-free), isolated, washed, resuspended in isolation buffer (IB; $50 \mathrm{mM}$ Tris pH 7.4, $150 \mathrm{mM} \mathrm{NaCl}, 1 \mathrm{mM}$ EGTA and $250 \mathrm{mM}$ sucrose) and homogenized in a glass Dounce homogenizer with 30 strokes of a Teflon pestle. The homogenate was spun twice at $800 \times g$ for $10 \mathrm{~min}$ to 
remove entire cells and nuclei, and the supernatant was centrifuged twice for $10 \mathrm{~min}$ at $8000 \times \mathrm{g}$. The resulting pellet (crude mitochondrial fraction) was collected, and the supernatant was centrifuged for an additional $30 \mathrm{~min}$ at $100,000 \times g$. The resulting pellet (LM fraction) and supernatant (cytosolic fraction) were centrifuged again at $100,000 \times g$ to further purify the fractions. The crude mitochondrial fraction was further purified by centrifugation twice at $8000 \times g$ for $10 \mathrm{~min}$. The obtained pellet was purified by centrifugation at $95,000 \times g$ for $30 \mathrm{~min}$ on a $30 \%$ Percoll density gradient in IB. The low-density band was centrifuged for $10 \mathrm{~min}$ at $7000 \times \mathrm{g}$, and the supernatant was centrifuged for $1 \mathrm{~h}$ at $100,000 \times g$ to obtain the MAM pellet. The high-density band was centrifuged twice for $10 \mathrm{~min}$ at $7000 \times \mathrm{g}$, and the resulting pellet contained the mitochondria. The obtained mitochondrial layer was washed free of Percoll and resuspended in IB. Isolation of mitochondria from MEF or U2OS cell lysates was performed with a Mitochondria Isolation Kit for Cultured Cells according to the manufacturer's protocol (Thermo Fisher Scientific, Inc.).

\section{Membrane fractionation assay and trypsin-protection assay}

For the membrane fractionation assay, the ER was isolated and resuspended in IB or IB containing $0.1 \mathrm{M} \mathrm{Na}_{2} \mathrm{CO}_{3}$ or $1 \%$ Triton X-100. After $15 \mathrm{~min}$ of incubation on ice, the samples were centrifuged at $20,000 \times g$ for $30 \mathrm{~min}$ to separate the pellet and soluble fractions. For the trypsin protection assay, the ER was incubated with $10 \mu \mathrm{g} / \mathrm{ml}$ trypsin (Promega) in the absence of protease inhibitors, and the reaction was terminated by the addition of PMSF to a final concentration of $2 \mathrm{mM}$.

\section{Immunoprecipitation and western blotting}

For immunoprecipitation experiments, nontransfected and transfected cells were lysed in whole-cell extraction buffer ( $10 \mathrm{mM}$ HEPES ( $\mathrm{pH}$ 7.9), $400 \mathrm{mM} \mathrm{NaCl}, 0.1 \mathrm{mM}$ EDTA, 5\% glycerol, $1 \mathrm{mM}$ DTT and protease inhibitors). Nontreated cell lysate or anti-CTRP1 $(1 \mu \mathrm{g})$ was added to the lysate, followed by the addition of anti-Flag-tagged agarose (Sigma) or protein A-agarose (Upstate Biotech) in TEG reaction buffer $(20 \mathrm{mM}$ Tris- $\mathrm{HCl}$ at $\mathrm{pH} 7.4,1 \mathrm{mM}$ EDTA, $10 \%$ glycerol, $1 \mathrm{mM}$ DTT and $150 \mathrm{mM}$ $\mathrm{NaCl}$ ), and the mixture was stirred for $3 \mathrm{~h}$ or overnight at $4{ }^{\circ} \mathrm{C}$ Immunoprecipitates were washed in TEG washing buffer (TEG reaction buffer containing $0.1 \%$ Triton-X 100). For Western blotting, cells were lysed in whole-cell extract buffer or homogenized using a MICCRA D-8 homogenizer (ART-moderne Labortechnik) in protein extraction buffer (20 mM HEPES (pH 7.9), $300 \mathrm{mM} \mathrm{NaCl}, 10 \mathrm{mM}$ EDTA, 0.1\% NP40, $100 \mathrm{mM}$ $\mathrm{KCl}$ and protease inhibitors). Total protein was fractionated on a sodium dodecyl sulfate polyacrylamide gel and transferred to nitrocellulose membranes (Amersham Biosciences). Primary antibodies against the following proteins were used: DRP1, cytochrome C, FAS (BD Biosciences), phospho-Drp1-Ser616 (Cell Signaling) BIP, calreticulin, calnexin, kinectin1, prohibitin, TOM20, PARP, ACSL4 (Santa Cruz Biotechnology), tubulin, calbindin, Flag (Sigma), CTRP1, Myc, cytochrome C, COX4 (Abcam) and caspase-3 (Cell Signaling). The secondary antibodies were fluoresceinconjugated anti-rabbit IgG, anti-mouse lgG, anti-goat IgG (Invitrogen) and HRP-conjugated antibodies to rabbit, mouse or goat antibody (Zymed Laboratories). Protein-antibody complexes were detected with the ECL Plus system (Amersham Biosciences).

\section{Live-imaging and confocal microscopy}

Live imaging of cells was performed with a spinning disc confocal system (A1C; Nikon). For live cells, imaging was performed at $37^{\circ} \mathrm{C}$ and $5 \% \mathrm{CO}_{2}$ in an LCl chamber (Chamlide TC; LCl), and images were taken with a $\times 60$ numerical aperture (NA) 1.4 oil objective lens. For fixed cells, imaging was performed with a laser-scanning confocal microscope (LSM780; Carl Zeiss). Images were taken with a $\times 63$ oil objective lens (NA 1.4). Images were analyzed using Carl Zeiss Elements, Photoshop (Adobe), IMARIS (Bitplane AG) or ImageJ (US National Institutes of Health; $\mathrm{NIH}$ ) software. Scale bars were generated using Carl Zeiss Elements and ImageJ.

\section{Mitochondrial length and DRP1 fluorescence analysis}

For measurement of mitochondrial length, the $z$-series stacks were imaged in increments of $0.2 \mu \mathrm{m}$ centered on the middle focal plane of the mitochondria. We analyzed the images of flat regions of cells with clearly compartmentalized mitochondria, and 20-30 mitochondria per cell were measured using Carl Zeiss Elements software. For analysis of DRP1 fluorescence, only mitochondria-associated DRP1 puncta on consecutive $z$ planes were quantified with ImageJ software $(\mathrm{NIH})$. Statistical analyses were performed using SigmaPlot (Systat Software, Inc.). Data are presented as the mean and s.e.m. or s.d. of at least three experiments.

\section{Immunofluorescence and histology}

Cultured cells were fixed in 4\% formaldehyde in PBS for 20 min at room temperature and, after being washed with PBS, were permeabilized with $0.1 \%$ Triton X-100 for 15 min. Cells were washed with PBS, blocked with $3 \%$ BSA in PBS for $1 \mathrm{~h}$ and incubated with primary antibodies in PBS for $\sim 1-3 \mathrm{~h}$ at room temperature. After being washed with PBS, the cells were incubated with secondary antibodies for $1 \mathrm{~h}$ at room temperature. The cells were then counterstained with $10 \mu \mathrm{M}$ 4,6-diamidino-2-phenylindole (DAPI) and mounted with Vectashield (VECTOR). Cryosections and paraffinembedded Section $(5-\mu \mathrm{m}$ thick) of the brain and liver were fixed in $10 \%$ buffered formaldehyde and used for H\&E staining, TUNEL assays, Nissl staining and immunofluorescence analyses.

\section{FAS-induced apoptosis}

Twelve-week-old Alb-Cre;Ctrp $1^{\mathrm{f} / \mathrm{fl}}$ and $C \operatorname{trp} 1^{\mathrm{f} / \mathrm{fl}}$ male mice were injected via the tail vein with an activating FAS-specific antibody (clone JO2; $0.25 \mu \mathrm{g} / \mathrm{g}$ body weight $(\mu \mathrm{g} / \mathrm{g})$ in sterile saline solution; BD Pharmingen) or with a sterile saline solution as a control ${ }^{35}$. After $24 \mathrm{~h}$, the indicated animals were euthanized for the analysis of apoptosis in the liver. Liver samples were fixed in $10 \%$ formalin for $24 \mathrm{~h}$, embedded in paraffin or optimal cutting temperature compound and sectioned to a $5-\mu \mathrm{m}$ thickness. Cryosections and paraffin-embedded sections were processed for H\&E staining, TUNEL assays and immunofluorescence analysis. For measurement of the plasma AST and ALT levels, fresh blood was collected from 4-h-fasted mice and centrifuged at $1500 \times g$ for $20 \mathrm{~min}$. The resulting supernatant (serum) was used to measure AST and ALT levels using a Hitachi autoanalyzer.

\section{Statistical analysis}

Differences between two experimental groups were analyzed using Student's $t$-test or the Mann-Whitney $U$ test. To compare three or more groups, we used one-way ANOVA with Šidák's correction. $P<0.05$ was considered statistically significant.

\section{RESULTS}

\section{CTRP1 is an ER membrane protein}

We first examined the subcellular localization and dynamic distribution of CTRP1 in various mouse and human cell lines. CTRP1 was distinctively localized to the ER, EMCSs and cytosol (Fig. $1 \mathrm{a}, \mathrm{b}$ and Supplementary Fig. $1 \mathrm{a}-\mathrm{g}$ ). Further analyses using ectopic expression of GFP-tagged CTRP1 showed that GFP-CTRP1 localized to the ER and to mitochondrial fission sites in living cells (Supplementary Fig. 1f). Electron microscopic (EM) analysis revealed that immunogold-conjugated CTRP1 localized to the mitochondria-associated ER membrane (MAM) (Fig. 1b and Supplementary Fig. 2). A previous paper suggested that CTRP1 has an $\mathrm{N}$-terminal signal peptide ${ }^{19}$, and our sequencing analysis of this $\mathrm{N}$-terminal signal peptide revealed that it contains a putative transmembrane domain (Supplementary Fig. 3a). To verify the function of this transmembrane domain, we expressed truncated versions of Myc-tagged CTRP1 in HeLa cells and examined their localization patterns (Supplementary Fig. 3c, d). We found that the CTRP1 mutants lacking the N-terminal domain localized to the cytosol, whereas those lacking the C-terminal $\mathrm{C} 1 \mathrm{q}$ domain localized to the ER (Supplementary Fig. $3 b$ and d). To further confirm the functionality of the $\mathrm{N}$-terminal transmembrane domain of CTRP1, we substituted the cysteine residue at position 13 of this domain with either alanine $\left(C T R P 1^{\mathrm{C} 13 \mathrm{~A}}\right)$ or arginine $\left(C T R P 1^{C 13 R}\right)$. Our results revealed that wild-type CTRP1 (CTRP1 ${ }^{\mathrm{WT}}$ ) and CTRP $1{ }^{\mathrm{C} 13 \mathrm{~A}}$ localized to the ER, whereas CTRP1 ${ }^{\mathrm{C} 13 \mathrm{R}}$ localized to the cytosol (Supplementary Fig. 3e). A fractionation assay using mouse embryonic fibroblasts (MEFs) revealed that CTRP1 was present in the cytosolic, ER and MAM fractions but not in the mitochondrial fraction (Fig. 1c). To further test whether CTRP1 is an ER membrane- or ER lumen-associated protein, we exposed ER fractions to high-pH or detergent conditions and then further 

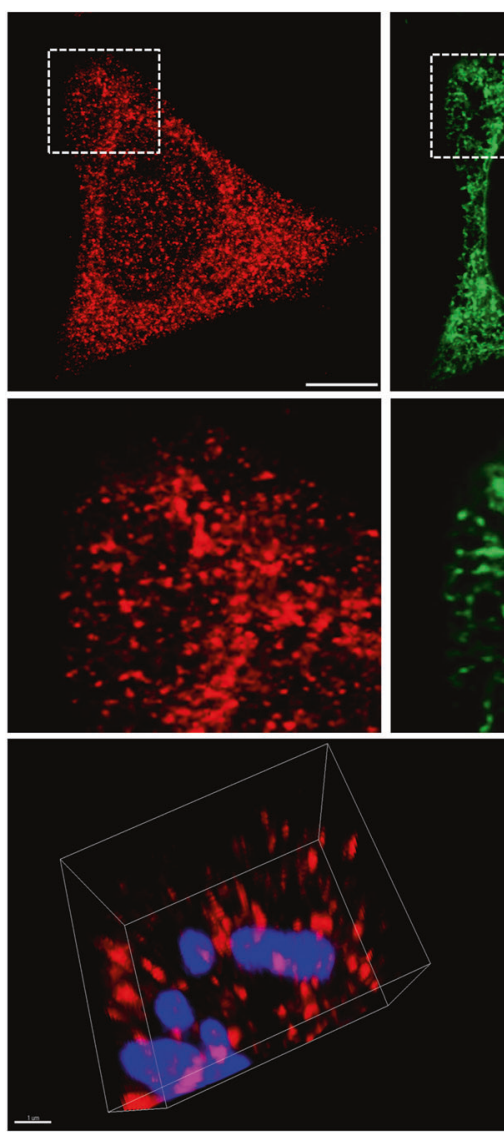

b

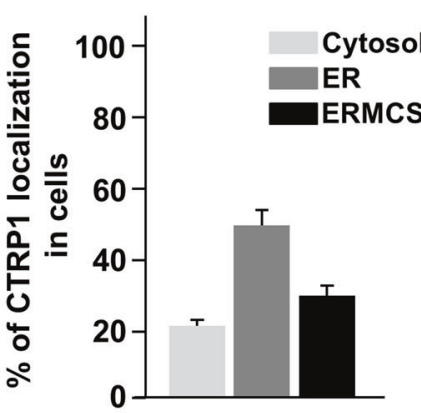

d

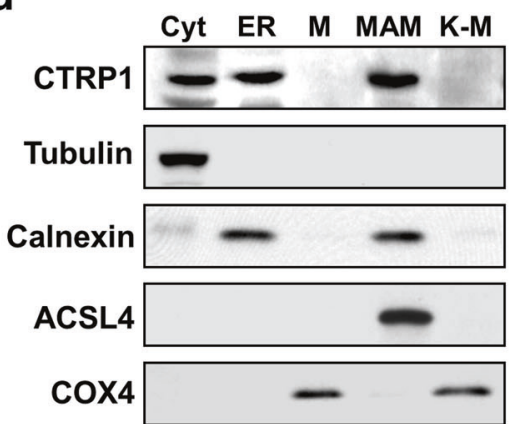

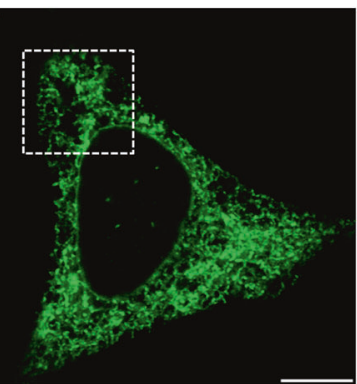
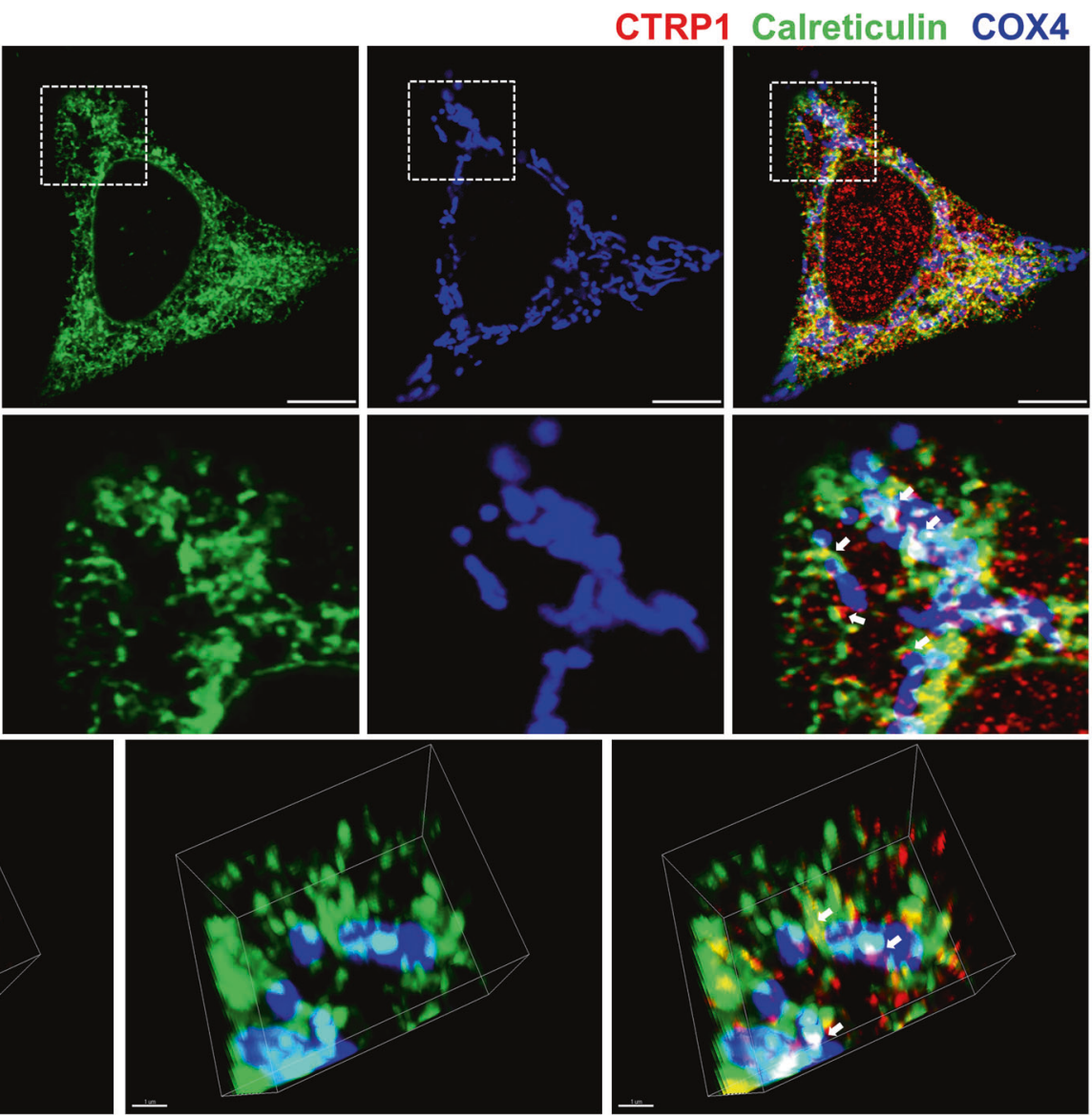

c
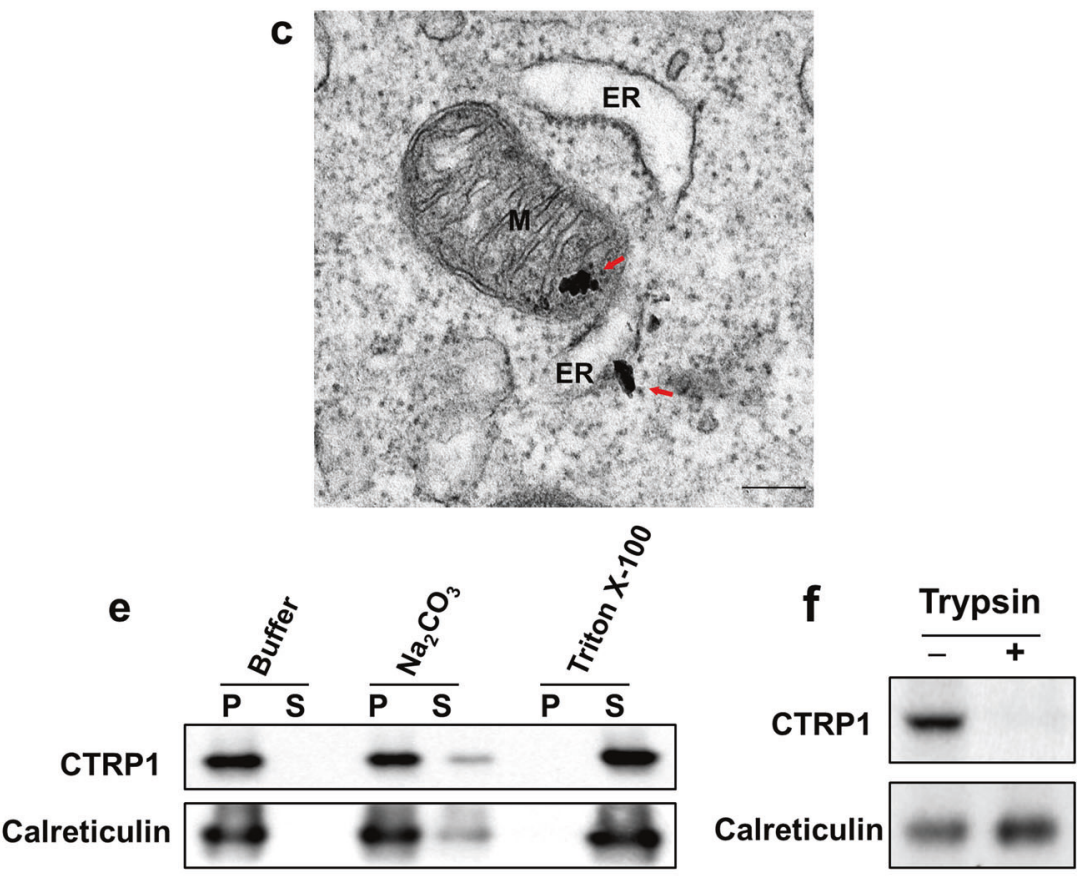

collected the pellet and supernatant fractions. We found that CTRP1 remained associated with the membrane at high $\mathrm{pH}$ but was partitioned into the soluble fraction in the presence of detergent (Fig. 1d). In addition, trypsin protection assays performed on the purified ER fraction revealed that CTRP1 was sensitive to trypsin treatment, whereas the ER lumen protein calreticulin was not (Fig. 1e, f). Taken together, these results indicate that CTRP1 is an ER membrane-bound protein that contains an $\mathrm{N}$-terminal transmembrane domain.

\section{CTRP1 regulates mitochondrial morphology}

To study the precise role of CTRP1 in specific cells and tissues, we generated conditional knockout mice in which exon 3 of Ctrp1 was deleted using the Cre/loxP system (Supplementary 
Fig. 1 CTRP1 is an ER membrane protein. a Representative low-magnification images (top), high-magnification images of the boxed areas (middle) and 3D reconstruction images (bottom) of mouse embryonic fibroblasts (MEFs) immunostained with CTRP1 (red), the ER marker calreticulin (green), and the mitochondrial protein COX4 (blue) antibodies. The white arrows indicate CTRP1 in the EMCSs. Scale bars, $10 \mu m$ (top) and $1 \mu \mathrm{m}$ (bottom). b Quantitation of CTRP1 localization to the cytosol, ER and ERMCSs in MEFs. c Representative electron micrograph of MEFs stained with anti-CTRP1 conjugated to 10-nm gold nanoparticles (red arrows). M mitochondrion, ER endoplasmic reticulum. Scale bar, $200 \mathrm{~nm}$. d Western blot analysis of subcellular fractions of MEFs. Cyt cytosol, MAM mitochondria-associated ER membrane, K-M Kit-purified mitochondria. The subcellular fractions were immunoblotted with antibodies against the cytosolic marker tubulin, the ER marker calnexin, the MAM marker ACSL4, and the mitochondrial marker COX4. e Western blot analysis of the pellet (P) and supernatant (S) fractions collected after ER samples were incubated with buffer, $\mathrm{Na}_{2} \mathrm{CO}_{3}$ or Triton X-100. f Western blot analysis of ER fractions that were treated with or without trypsin. Throughout, the data are presented as the mean \pm s.e.m.

Fig. 4). We first transfected embryos harboring loxP-flanked (floxed) Ctrp $1\left(C \operatorname{trp} 1^{\mathrm{fl} / \mathrm{fl}}\right)$ with a lentivirus encoding the Cre recombinase and collected MEFs for analysis (Supplementary Fig. 4a-d). The $\mathrm{Cre}^{+} \mathrm{Ctrp}^{\mathrm{fl/fl}}\left(\mathrm{Ctrp}^{-/-}\right)$MEFs showed elongated mitochondria (Fig. 2a and Supplementary Fig. 5a), which are a characteristic sign of a fission defect, but did not show any morphological change in the ER or Golgi apparatus (Supplementary Fig. 6). The mitochondrial length was significantly greater in the $\mathrm{Cre}^{+} \mathrm{Ctrp} 1^{\mathrm{f} / \mathrm{fl}}$ MEFs than in the $\mathrm{Cre}^{-} \mathrm{Ctrp} 1^{\mathrm{f} / \mathrm{fl}} \mathrm{MEFs}$ that did not express Cre (Ctrp1 ${ }^{+/+}$MEFs) (Fig. 2b). Next, we used siRNAs to deplete DRP1, MFF, Mid51 or CTRP1 from human bone osteosarcoma (U2OS) cells (Supplementary Fig. 5c). DRP1 is critical to the induction of mitochondrial fission, and its adaptors, MFF and MiD51, regulate mitochondrial fission by recruiting DRP1 in mitochondria ${ }^{23-26}$. Consistent with our observations in the $\mathrm{Cre}^{+} \mathrm{Ctrp} 1^{\mathrm{f} / \mathrm{fl}} \mathrm{MEFs}$, we found that depletion of CTRP1 from U2OS cells significantly altered the mitochondrial morphology, similar to mitochondrial morphology defects following depletion of DRP1, MFF or MIEF1 (also known as MiD51) from U2OS cells (Fig. 2c, d and Supplementary Fig. 5b). The mitochondrial morphology defects in the U2OS cells treated with a CTRP1-specific siRNA (siCTRP1) were substantially rescued by overexpression of CTRP1 (Supplementary Fig. 7). We also analyzed the mitochondrial dynamics in the HeLa cells cotranfected with mito-RFP and mito-PAGFP after either control or CTRP1 siRNA treatment and found that CTRP1 was not associated with mitochondrial fusion (Supplementary Fig. 8). These results suggest that CTRP1 is involved in mitochondrial fission.

\section{CTRP1 interacts with DRP1}

To investigate the function of ER-bound CTRP1 in mitochondrial fission, we first analyzed the subcellular localizations of Ctrp1 and Drp1 in MEFs and found that endogenous Ctrp1 partially colocalized with Drp1 at the EMCSs (Fig. 3a and Supplementary Fig. 1g). Next, we used immunoprecipitation (IP) assays to test for a physical interaction between Flag-CTRP1 and GFP-DRP1, between Flag-CTRP1 and endogenous DRP1 or between endogenous CTRP1 and DRP1. Our results confirmed that CTRP1 binds to DRP1 (Fig. 3b-d). To determine which CTRP1 domains interact with DRP1, we examined the physical interaction between Flag-CTRP1 truncated mutants and GFP-DRP1. We found that the C1q domain of CTRP1 interacts with DRP1 (Supplementary Fig. 9a). This finding suggests that the interaction between ER-bound CTRP1 and mitochondrial DRP1 may be required for mitochondrial fission events in the EMCSs. We also observed partial colocalization and physical interaction of CTRP1 with the mitochondrial DRP1 adaptor proteins MIEF2 (also known as MiD49) and MiD51 (Supplementary Fig. 9b, c). Additionally, we found that Mid49 or Mid51 did not affect the physical interaction between Flag-CTRP1 and GFP-DRP1 (Supplementary Fig. 9d). These findings suggest that the ER-mitochondrion interface might be necessary for mitochondrial fission. Moreover, our results collectively indicate that CTRP1 is an ER membrane protein that interacts with DRP1 at the EMCSs.

\section{CTRP1 recruits DRP1 and acts as a DRP1 adaptor}

As DRP1-interacting mitochondrial proteins influence the recruitment of DRP1 in mitochondria, we next analyzed the effects of CTRP1 on DRP1 recruitment to determine the role of CTRP1 in mitochondrial fission. The fluorescence intensity of the Drp1 signal was decreased in the mitochondria of the Cre ${ }^{+} \mathrm{Ctrp} 1^{\mathrm{f} / \mathrm{fl}} \mathrm{MEFs}$ relative to those in the $\mathrm{Cre}^{-} \mathrm{Ctrp} 1^{\mathrm{fl} / \mathrm{fl}}$ MEFs (Fig. 4a). To investigate whether Ctrp1 deficiency affects the Drp1 protein levels in mitochondria, we examined the protein levels of Drp1 and found that they were decreased in the mitochondria of the Cre ${ }^{+} C \operatorname{Ctp} 1^{\mathrm{f} / \mathrm{fl}}$ MEFs (Fig. 4b). We generated Alb-Cre;Ctrp $1^{\mathrm{f} / \mathrm{fl}}$ mice and confirmed that Ctrp1 was abolished in the Alb-Cre;Ctrp $1^{\text {fl/fl }}$ liver cells (Fig. 4c) and further found that Drp1 protein levels were decreased in the mitochondria of the Alb-Cre;Ctrp $1^{\text {fl/fl }}$ liver cells (Fig. 4d). Consistent with these findings, depletion of CTRP1 in U2OS cells significantly altered the mitochondrial localization of the DRP1 signal, whereas depletion of MFF or MiD51 had no such effect (Fig. 4e). CTRP1 depletion also resulted in a significant decrease in the DRP1 protein levels (Fig. 4f), indicating that CTRP1 is involved in the recruitment of DRP1. However, CTRP1 did not alter the phosphoDrp1 levels (Supplementary Fig. 9e). Collectively, these results suggest that ER-bound CTRP1 interacts with DRP1, acts as a DRP1 adaptor and regulates mitochondrial fission events via its effects on DRP1 recruitment.

\section{Ablation of CTRP1 leads to apoptotic resistance}

DRP1 influences the oligomerization of the proapoptotic protein BAX through mitochondrial membrane remodeling and is thereby involved in apoptotic resistance ${ }^{30}$. As mammalian cells undergo apoptosis, many proteins, including cytochrome $\mathrm{c}$ in the mitochondrial intermembrane space, are forced out from the mitochondria when the permeability of the mitochondrial outer membrane (MOM) is increased via pores formed by the proapoptotic BCL-2 family members $B A X$ and $B A K^{31,32}$. Depletion of $B A X$ or BAK is associated with apoptotic resistance, and the mitochondrial DRP1 adaptor proteins that regulate the recruitment and oligomerization of DRP1 have also been implicated in resistance to cell death ${ }^{25,26}$. Accordingly, we next investigated the potential function of CTRP1 in apoptosis by examining whether CTRP1 depletion affected the cell death induced by staurosporine (STS), actinomycin D or etoposide. Normal U2OS cells had filamentous mitochondria that maintained their cytochrome $\mathrm{c}$ under normal conditions, whereas the STS-treated U2OS cells showed fragmented mitochondria and the release of cytochrome $c$ into the cytosol (Supplementary Fig. 10a, b). Activated caspase-3 (Supplementary Fig. 10c) and caspase-3-mediated cleavage of PARP (Supplementary Fig. $10 \mathrm{~d})$ were detected in the STS-treated U2OS cells. To elucidate the potential function of CTRP1 in apoptotic resistance, we examined cytochrome $c$ release in the siCTRP1-treated U2OS cells. Compared to the control siRNA-treated U2OS cells, the CTRP1-depleted cells exhibited decreased cytochrome $c$ release similar to that observed in the DRP1-, BAX- and BAK-ablated cells (Fig. $5 \mathrm{a}, \mathrm{b}$ ). Caspase- 3 activation and PARP cleavage were also decreased in the CTRP1-depleted cells (Fig. 5c). Finally, we 


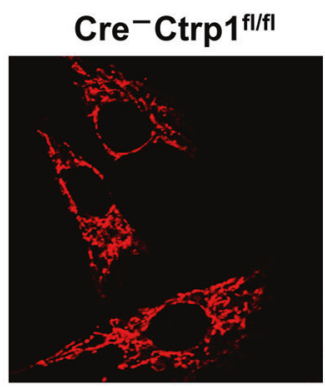

Cre ${ }^{+}$Ctrp1 ${ }^{\text {fl/fl }}$

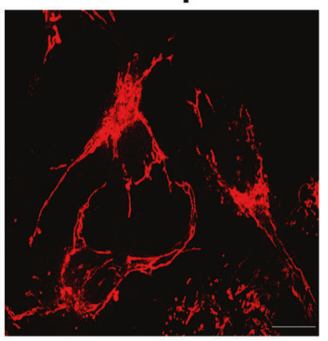

C

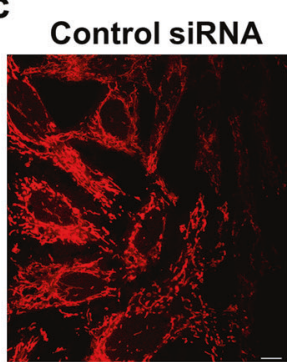

b

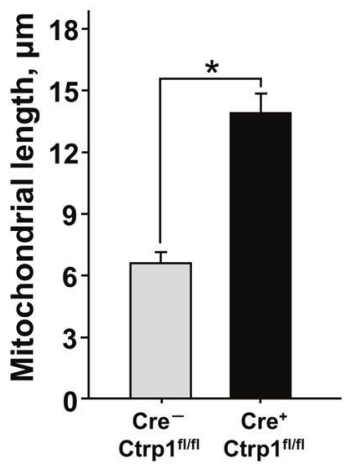

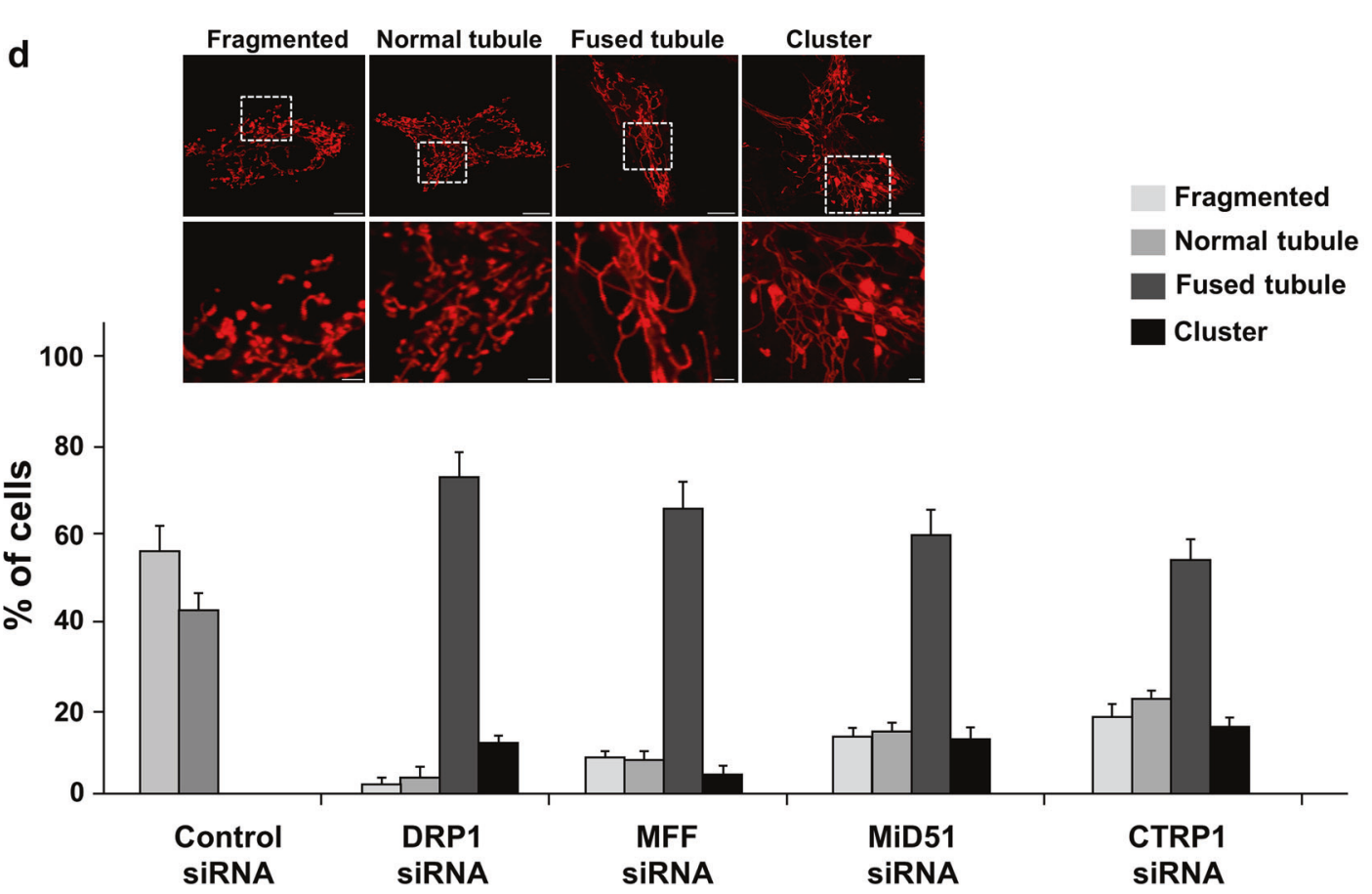

Fig. 2 CTRP1 regulates mitochondrial morphology. a MitoTracker-stained $\mathrm{Cre}^{-} \mathrm{Ctrp} 1^{\mathrm{fl} / \mathrm{fl}}$ and $\mathrm{Cre}^{+} \mathrm{Ctrp} 1^{\mathrm{fl} / \mathrm{fl}} \mathrm{MEFs}$. b Quantification of mitochondrial lengths in Cre ${ }^{-} \mathrm{Ctrp} 1 \mathrm{fl/fl}$ and Cre ${ }^{+} \mathrm{Ctrp} 1 \mathrm{fl} / \mathrm{fl}$ MEFs ( $n=256-427$ mitochondria of $30-50$ cells). c and d MitoTracker staining (c) and quantification of mitochondrial morphology (d) in human bone osteosarcoma (U2OS) cells transfected with the indicated siRNAs $(n=300$ cells). Quantitative data were obtained by analysis of random samples and are presented as the mean \pm s.e.m. of three independent experiments ${ }^{*} P<0.001$. Scale bars, $10 \mu \mathrm{m}$ in (a) and (c), $10 \mu \mathrm{m}$ (top) and $2 \mu \mathrm{m}$ (bottom) in (d).

analyzed whether CTRP1 depletion affected the survival of cells treated with actinomycin D or etoposide. Indeed, our quantification analyses showed that the number of apoptotic cells was decreased in the CTRP1-ablated cell cultures treated with these agents (Fig. 5d). Apoptotic resistance, such as caspase-3 activation, PARP cleavage, cytochrome $c$ release and apoptotic HeLa cells after treatment with a CTRP1-specific siRNA (siCTRP1), was generally rescued by overexpression of CTRP1 (Supplementary Fig. $10 \mathrm{e}-\mathrm{g}$ ). Our results therefore suggest that CTRP1 plays an important role in the ability of cells to resist apoptosis.

\section{Ctrp1 depletion contributes to Fas-induced apoptotic} resistance in liver cells and degeneration of neuronal cells FAS-induced cell death in the liver is known to depend on mitochondria $^{36,37}$. To investigate whether Ctrp1 contributes to regulating apoptosis in vivo, we induced apoptotic liver damage in the Alb-Cre;Ctrp $1^{\mathrm{fl} / \mathrm{fl}}$ and control $C \operatorname{trp} 1^{\mathrm{f} / \mathrm{fl}}$ mice by tail vein injection of FAS-specific antibodies. We then examined the serum levels of aspartate transaminase (AST) and alanine transaminase (ALT), which are indicators of liver damage, at $24 \mathrm{~h}$ post-injection. The anti-FAS-treated $A / b-C r e ; C \operatorname{trp} 1^{\mathrm{fl} / \mathrm{fl}}$ mice exhibited significantly 
a
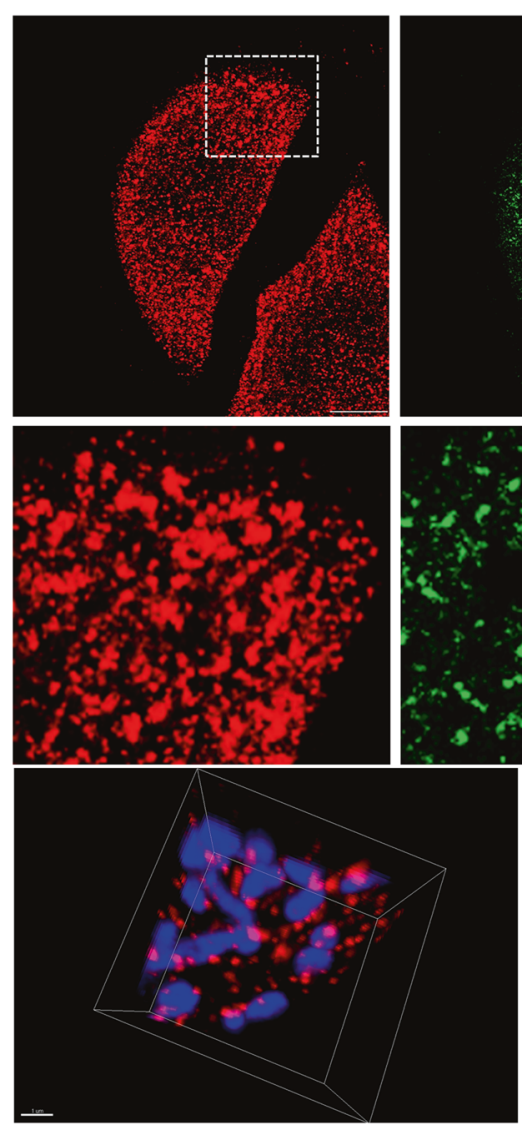

b

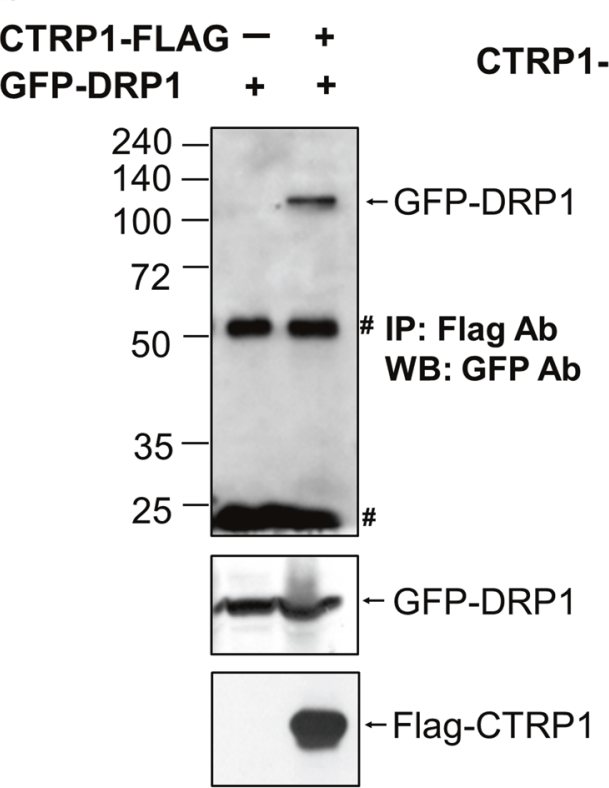

CTRP1 DRP1COX4
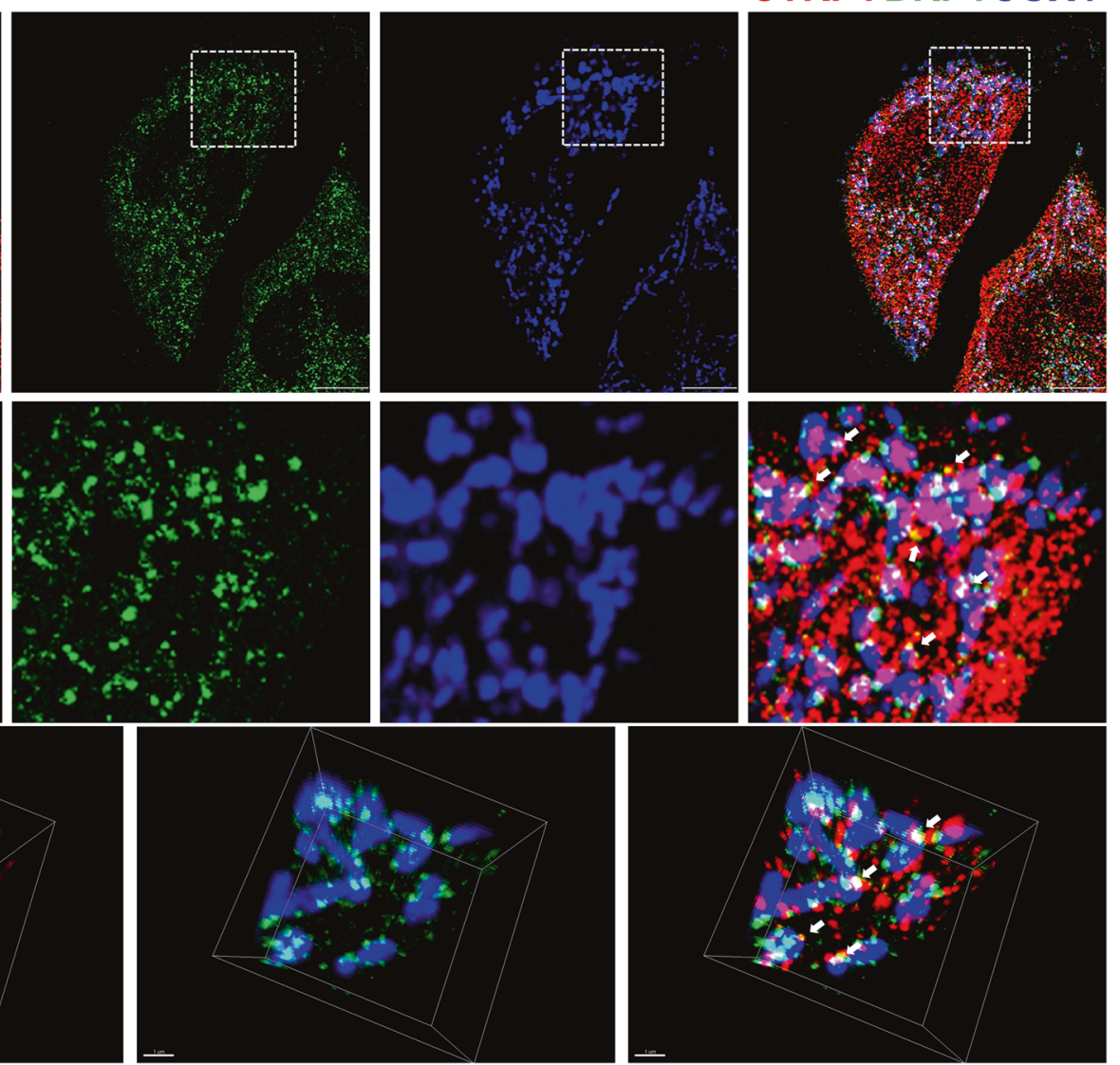

IP:

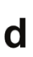

d

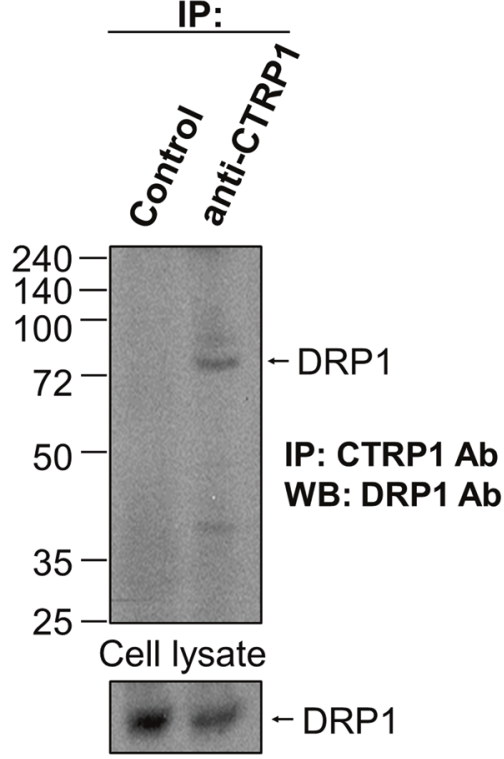

Fig. 3 CTRP1 interacts with DRP1. a Representative low-magnification images (top), high-magnification images of the boxed areas (middle) and 3D reconstruction images (bottom) of MEFs immunostained with antibodies against CTRP1 (red) and DRP1 (green) and the mitochondrial protein COX4 (blue). The white arrows indicate colocalization of CTRP1 and DRP1. Scale bars, $1 \mu \mathrm{m}$ (top) and $10 \mu \mathrm{m}$ (bottom). b Whole-cell lysates (WCLs) of 293T cells cotransfected with constructs expressing Flag-CTRP1 and GFP-DRP1 were immunoprecipitated (IP) with anti-Flag beads and then immunoblotted (IB) with a GFP-specific antibody. Asterisks indicate immunoglobulin G (IgG). c Interaction between endogenous DRP1 and Flag-CTRP1; 293T cells were transfected with Flag-vector (Lane 1) or Flag-CTRP1 (Lane 2), and WCLs were immunoprecipitated with anti-Flag beads and blotted with anti-DRP1. d Interaction between endogenous DRP1 and CTRP1. WCLs of 293T cells were immunoprecipitated with anti-CTRP1 and blotted with anti-Drp1. 


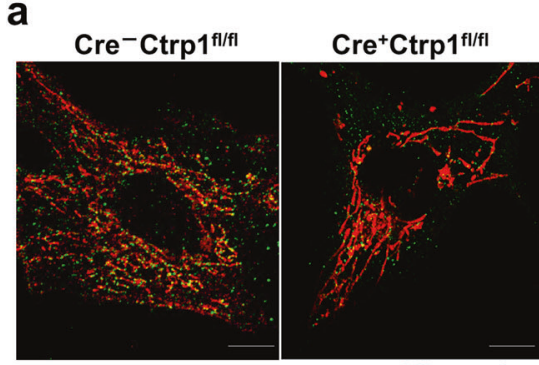

Drp1 Mitotracker

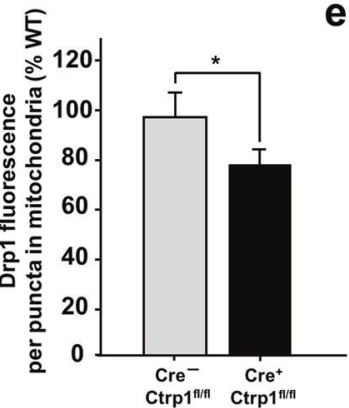

b

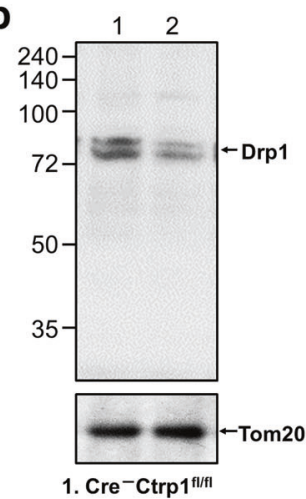

2. Cre + Ctrp 1 fl/fil

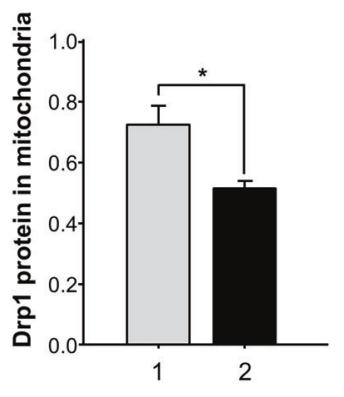

d

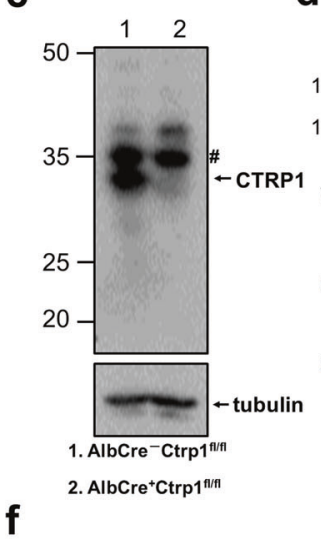

d

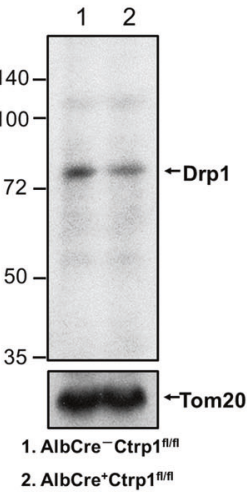

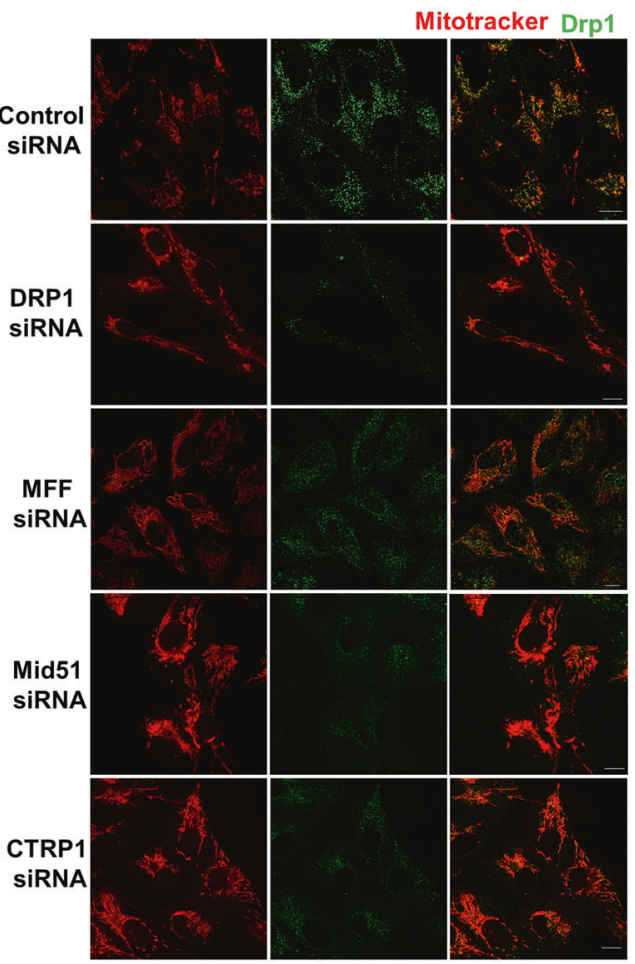

$$
\sum_{3}
$$
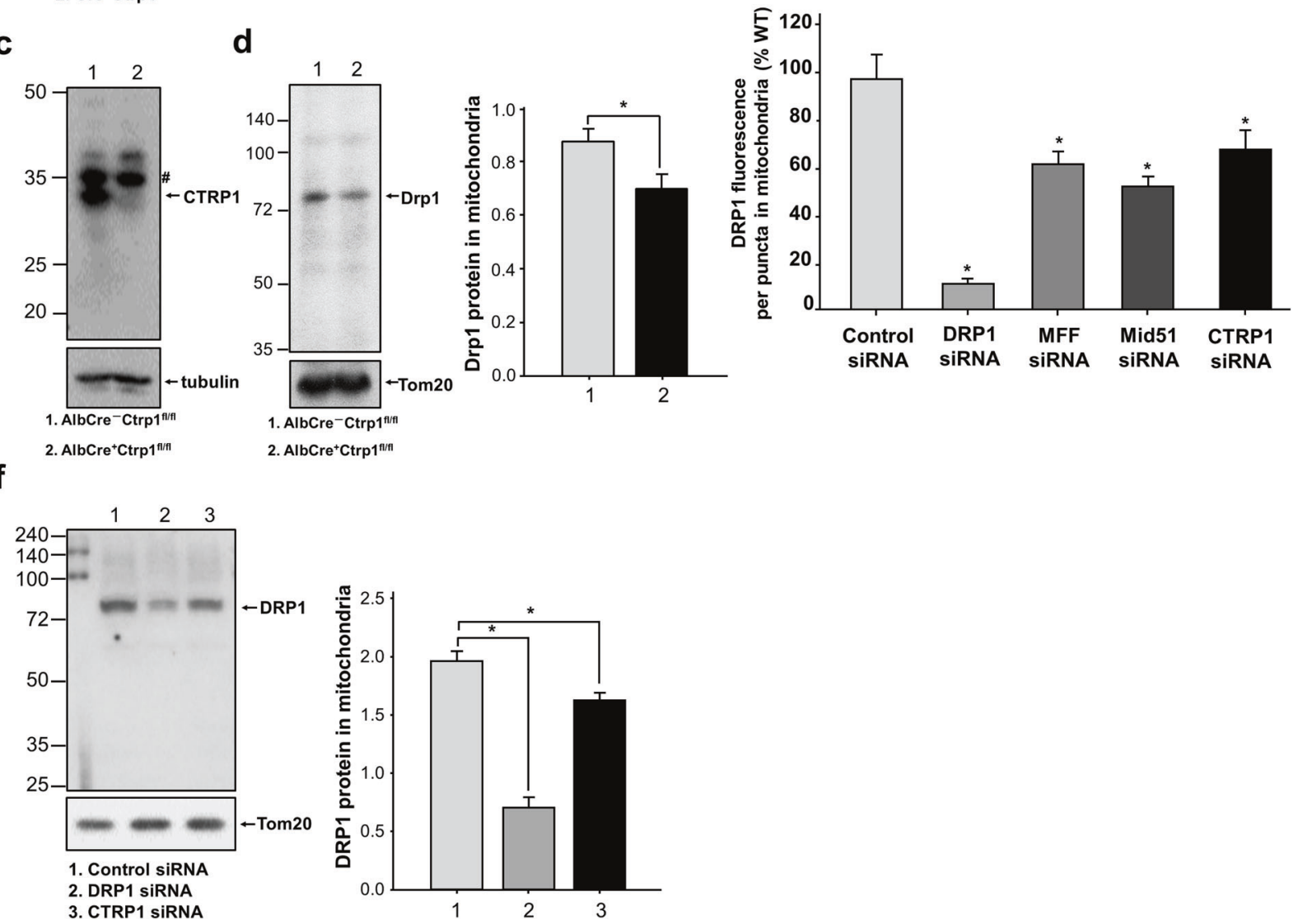

Fig. 4 CTRP1 regulates the recruitment of DRP1 during mitochondrial fission. a and e MitoTracker and anti-Drp1 staining of the indicated cells. Quantification of the Drp1 fluorescence signal from three independent biological replicates is shown. $\mathbf{b}$, $\mathbf{d}$ and $\mathbf{f}$ Immunoblotting of mitochondrial fractions and WCLs with antibodies against DRP1 and the mitochondrial protein Tom20. c Immunoblotting of the Alb$\mathrm{Cre}^{-} \mathrm{Ctrp} 1^{f / f}$ and $\mathrm{Alb}-\mathrm{Cre}{ }^{+} \mathrm{Ctrp} 1^{f / / f l}$ cells with antibodies against CTRP1 and the cytosolic marker tubulin. The asterisks in $\mathbf{f}$ represent nonspecific bands. Data are presented as the mean and s.e.m. ${ }^{*} P<0.001$. Scale bars, $10 \mu \mathrm{m}(\mathbf{a})$ and (e). 

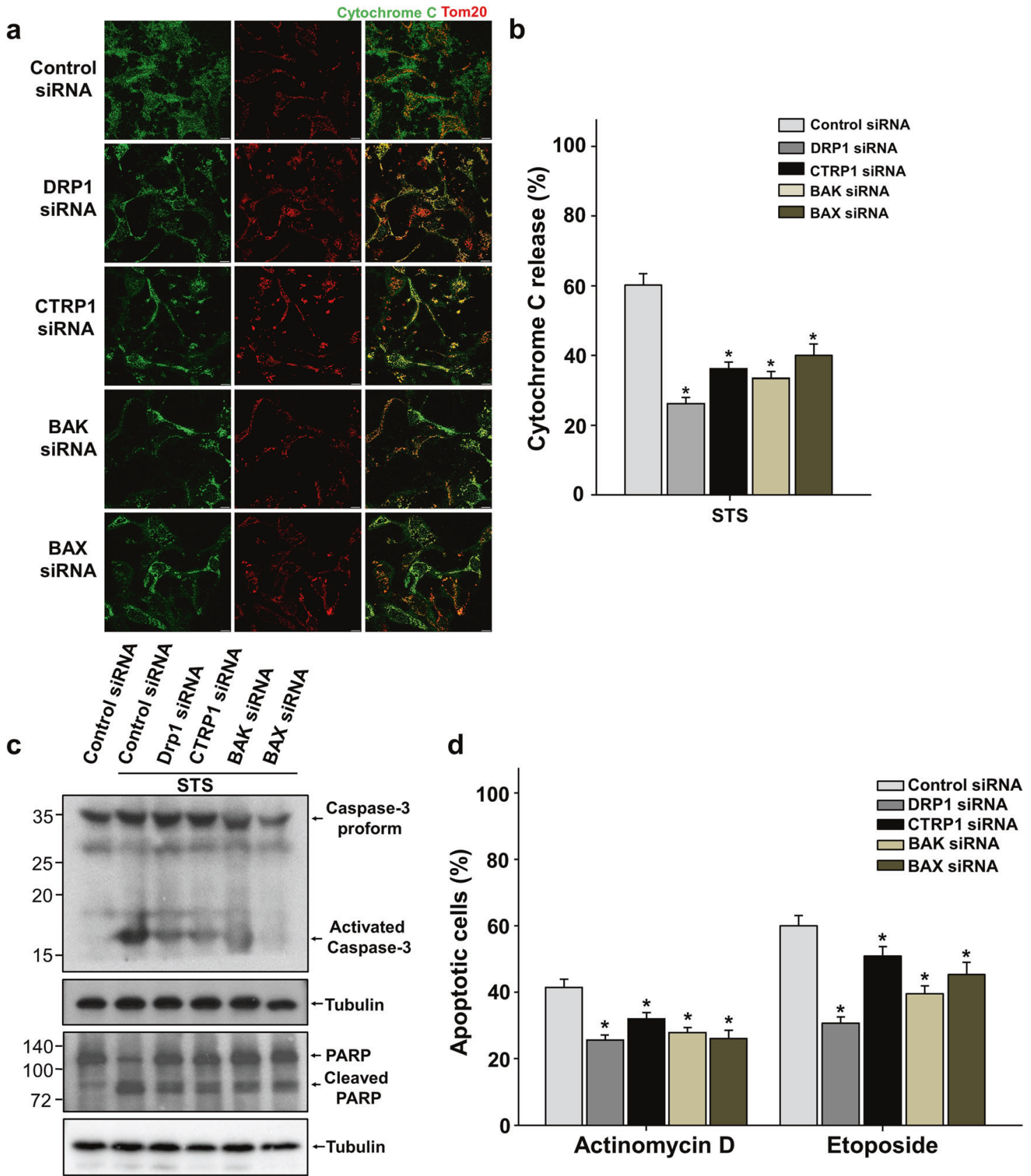

Fig. 5 CTRP1-deficient cells are less sensitive to the induction of apoptosis. a-c Apoptotic analysis of U2OS cells transfected with the indicated siRNAs and treated with STS, MitoTracker (red) and anti-cytochrome C antibody (green) staining (a), quantification of cytochrome C release ( $n=600$ cells) (b) and immunoblotting with caspase-3, PARP and the cytosolic marker tubulin antibodies (c). d Quantification of fragmented nuclei in cells treated with actinomycin D or etoposide ( $n=600$ cells). Data are presented as the mean and s.e.m. Scale bars, $10 \mu \mathrm{m}$ in (a).

smaller increases in AST and ALT levels than the anti-FAS-treated control $C \operatorname{trp} 1^{\mathrm{fl} / \mathrm{fl}}$ mice (Fig. 6a), and their livers showed a more intact lobular architecture and lower levels of hemorrhagic infiltrates than those of the control mice (Fig. 6b). TUNEL assays revealed that the livers of the anti-FAS-treated Alb-Cre;Ctrp $1^{\mathrm{f} / \mathrm{fl}}$ mice exhibited less apoptotic cell death than those of the antiFAS-treated $\mathrm{Ctrp}^{\mathrm{fl} / \mathrm{fl}}$ mice (Fig. 6c). An analysis of subcellular cytochrome $\mathrm{c}$ distribution showed that less cytochrome $\mathrm{c}$ was released from mitochondria in the livers of the anti-FAS-treated $\mathrm{Alb}-\mathrm{Cre} ; \mathrm{Ctrp}^{\mathrm{fl} / \mathrm{fl}}$ mice than in those of the anti-FAS-treated Ctrp $1^{\mathrm{f} / \mathrm{fl}}$ mice (Fig. $6 \mathrm{~d}$ ). These results collectively suggest that Ctrp1 plays a crucial role in the ability of hepatocytes to resist apoptosis in vivo. Finally, since alterations in the genes involved in mitochondrial fission and fusion lead to neuronal dysfunction, which can result in the degeneration of Purkinje cells (PCs) in the cerebellum ${ }^{38,39}$, we examined whether Ctrp1 deletion could trigger neuronal degeneration in Pcp2-Cre;Ctrp $1^{\mathrm{f} / \mathrm{fl}}$ mice, in which Ctrp1 is specifically knocked out in PCs. Indeed, the number of cerebellar PCs in the Pcp2-Cre;Ctrp $1^{\text {fl/fl }}$ mice was much lower than that in the Ctrp $1^{1 / / 1}$ mice (Fig. 6e, f), suggesting that Ctrp1 is required for neuronal cell survival.

\section{DISCUSSION}

We show that CTRP1 is a novel ER membrane protein that interacts with the mitochondrial fission-related proteins DRP1, 


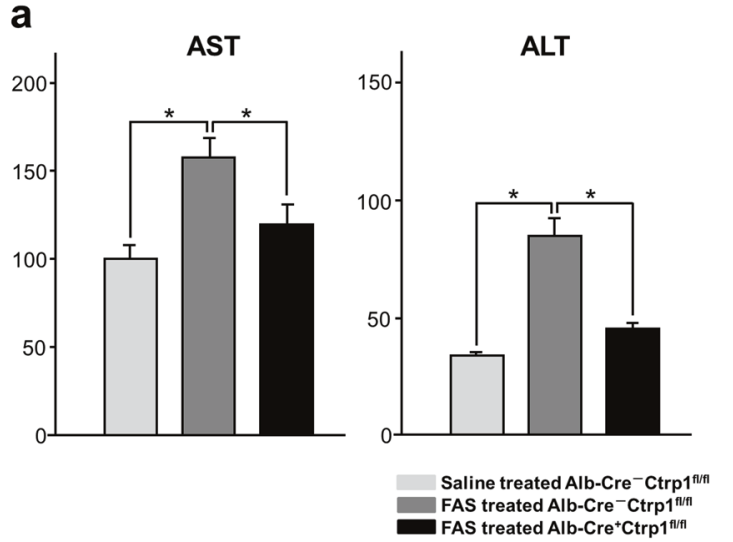

C
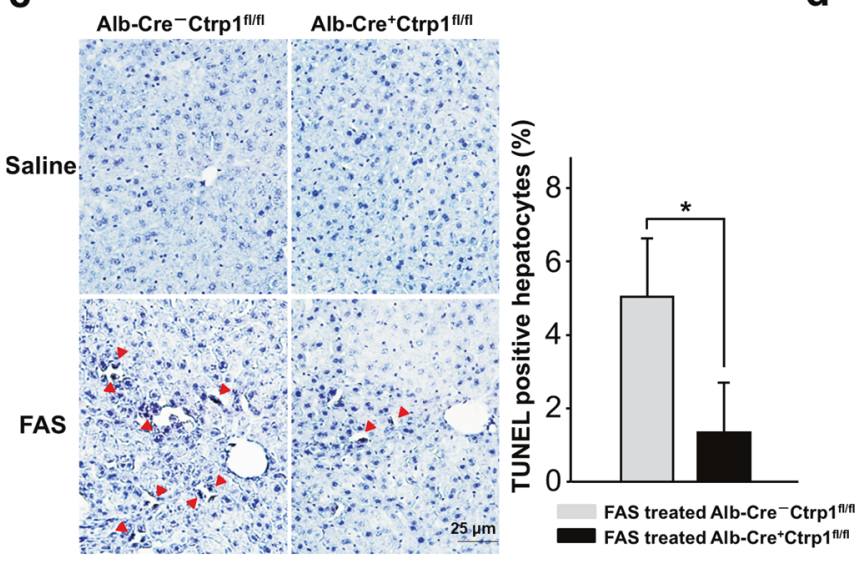

e

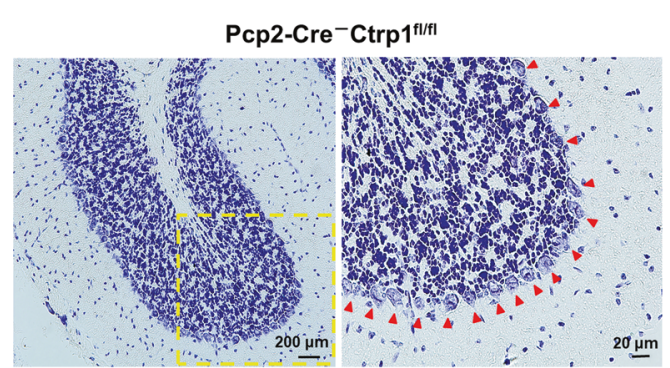

Pcp2-Cre+Ctrp1 $1^{\text {fl/fI }}$

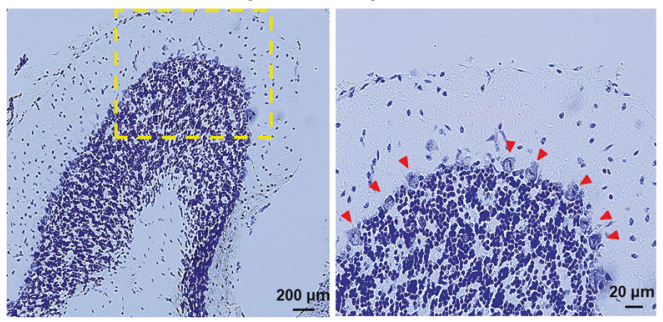

FAS

d

f b
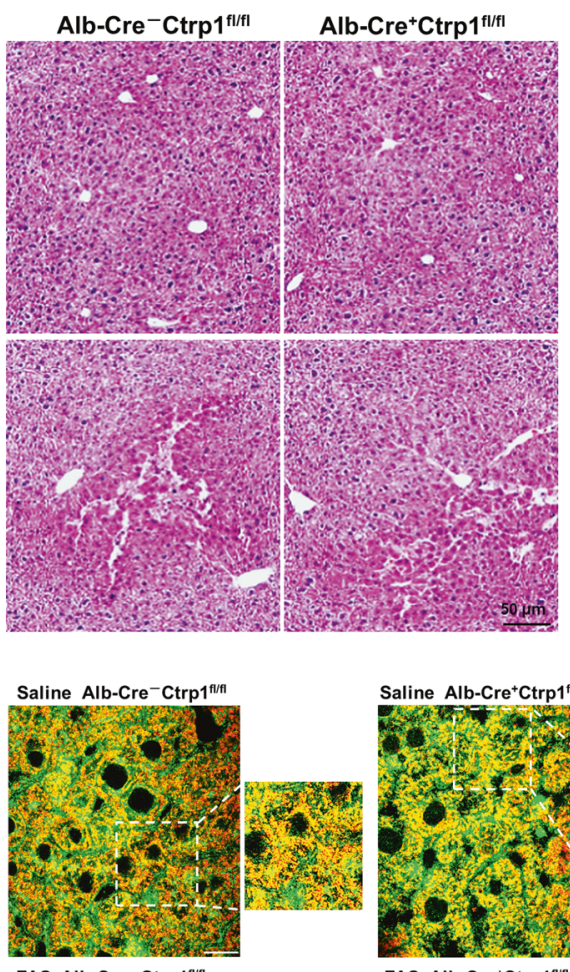

Saline Alb-Cre+Ctrp1 $1^{\text {flf }}$

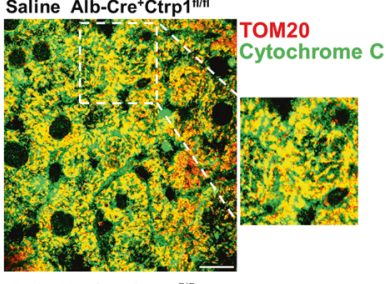

FAS Alb-Cre-Ctrp1"1/til

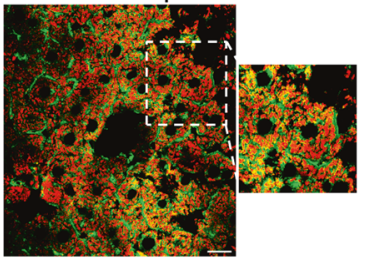

FAS Alb-Cre+Ctrp1 ${ }^{\text {fluf }}$
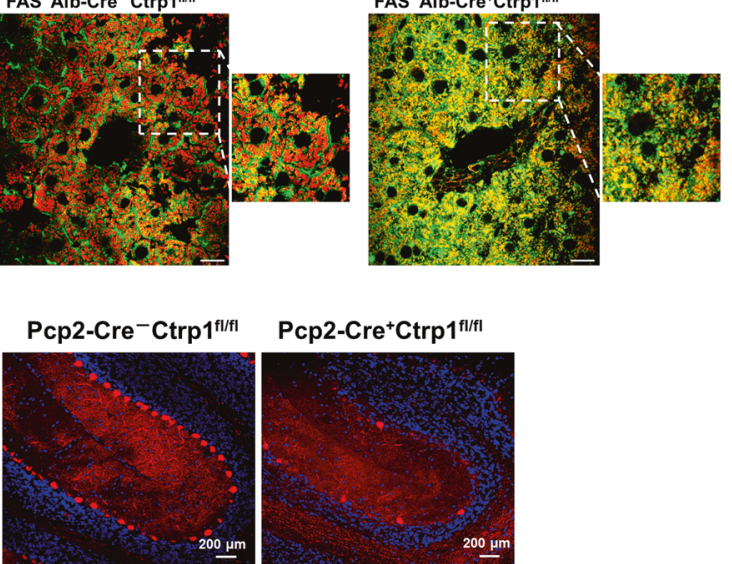

Pcp2-Cre ${ }^{+}$Ctrp1 $1^{\text {fl/fl }}$
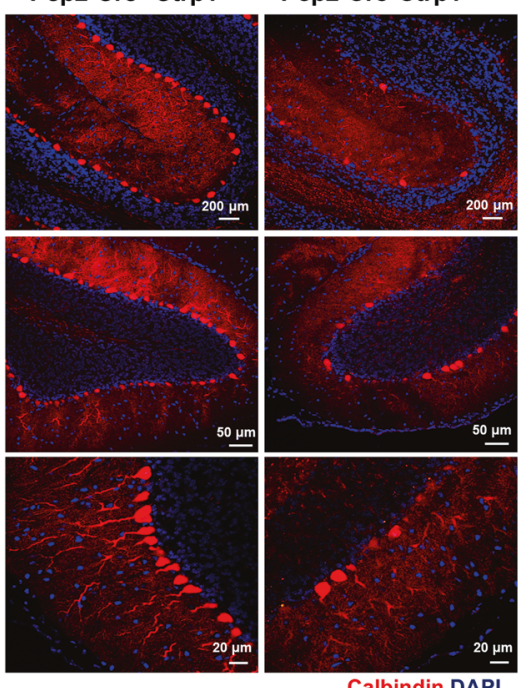

Fig. 6 Ctrp1 deficiency causes resistance to Fas-induced apoptosis and triggers neuronal defects. a-d Plasma AST and ALT levels (a), hematoxylin and eosin (H\&E) staining (b), TUNEL staining (c) and immunostaining with antibodies against cytochrome $C$ (green) and Tom 20 (red) (d) in liver sections obtained $24 \mathrm{~h}$ after 3-month-old mice were injected with anti-Fas (Fas) or saline, as indicated. e and $\mathbf{f}$ Nissl staining (e) and immunostaining for the Purkinje cell (PC) marker calbindin (f) in the cerebella of 10-week-old mice injected as indicated. Data are presented as the mean and s.e.m. Scale bars are as indicated.

MiD49 and MiD51 at the mitochondrial fission site (Supplementary Fig. 12). Mitochondrial fission is centrally regulated by Drp1, which oligomerizes around mitochondrial constriction sites and can drive mitochondrial fission. At a mitochondrial constriction site, the GTPase dynamin 1 (dnm1 in yeast) and Drp1 oligomerize into a helical ring that is much smaller than the diameter of the mitochondrion ${ }^{40-44}$. Thus, certain Drp1-associated factors may help Dnm1 and Drp1 drive mitochondrial constriction. If this is the 
case, such factors could be necessary for mitochondrial division. Our findings indicate that the interaction between ER-bound CTRP1 and Drp1 is required to regulate mitochondrial morphology in EMCSs. Additionally, our data suggest that ER-bound CTRP1 may regulate the recruitment of DRP1 by cooperating with MiD49 and MiD51, which are known DRP1 adaptors.

Previous studies have focused on the function of secreted CTRP1, which has been shown to regulate metabolic disease and cardiovascular function ${ }^{1-13}$. In the present study, we identified CTRP1 as an ER membrane protein that localizes to the EMCSs and regulates mitochondrial fission. Interestingly, immunoblot analysis of N-terminal or C-terminal GFP-tagged CTRP1 indicated that CTRP1 can have membrane-bound and secreted forms (Supplementary Fig. 11). The Peptide Cutter and PROSPER programs predicted that CTRP1 could have potential cleavage sites in its short variable domain (data not shown), adding weight to the idea that CTRP1 has both secreted and membrane-bound forms. Based on the existing evidence and our present findings, we speculate that CTRP1 could have a dual function, wherein its secreted form regulates metabolic and cardiovascular functions while its ERbound form regulates mitochondrial fission. Given that mitochondrial dynamics are also critical for metabolic and cardiovascular functions ${ }^{45}$, it is necessary to further investigate whether the secreted form of CTRP1 can indirectly modulate mitochondrial dynamics.

ER tubules are wrapped around mitochondrial constriction sites and fission sites ${ }^{27}$. The ER-mitochondrion connection is mediated by the ER-mitochondria encounter structure (ERMES) complex in yeast and by the mitochondrial fusion protein mitofusin 2 (MFN2) in mammalian cells, both of which tether the ER to mitochondria in their respective cells ${ }^{46,47}$. Interestingly, depletion of the ERMES complex triggers a mitochondrial fission defect, whereas MFN2 deficiency leads to a mitochondrial fusion defect ${ }^{39}$. This finding raises the possibility that, reminiscent of the ERMES complex in yeast, the ER of mammalian cells may contain yet-unknown fission-associated factors beyond MFN2. Here, we provide multiple lines of evidence showing that ER-associated CTRP1 participates in mitochondrial fission events via an interaction with the mitochondrial fission protein DRP1.

The DRP1 adaptor proteins MFF, MiD49 and MiD51 form contacts with the ER in the EMCSs ${ }^{27,48}$. Recently, Osellame et al. investigated proteins that interact with MFF, MiD49 or MiD51 in the EMCSs ${ }^{26}$. These researchers showed that both MFF and MiD51 bound DRP1 but not mitochondrial Spire1 or the ER membrane proteins INF2 and Syn17, which are known to support mitochondrial constriction through actin polymerization ${ }^{28,29,49}$. This finding suggested that ER-bound proteins can interact with MFF, MiD49 and MiD51 or with DRP1 in the MAM. Interestingly, Ji et al. suggested that ER-bound MFF can mediate DRP1 oligomerization and that ER-associated DRP1 regulates mitochondrial fission ${ }^{50}$. Thus, further studies should investigate whether ER-bound CTRP1 contributes to DRP1 oligomerization. Here, we showed that CTRP1 is an ER-associated DRP1 adaptor that recruits DRP1 during mitochondrial fission. This recruitment of DRP1 could be necessary for the interaction with the mitochondrial DRP1 adaptors MFF, MiD49 and MiD51 and/or the ER-associated DRP1 adaptor CTRP1. Taken together, our findings and those in the literature suggest that complex networks of mitochondrial and ER-associated DRP1 adaptors critically contribute to mitochondrial fission by facilitating the recruitment and maturation of DRP1.

Studies have shown that during apoptosis, DRP1 affects Bax oligomerization via mitochondrial membrane remodeling ${ }^{29}$ and that ablation of the mitochondrial DRP1 adaptors MiD49 and MiD51 protects cells against apoptosis by modulating mitochondrial crista remodeling ${ }^{25}$. These results suggest that defective recruitment and/or assembly of DRP1 causes apoptotic resistance. Here, we showed that CTRP1 inactivation triggers resistance to FAS-induced apoptosis in liver cells. Thus, our results indicated that ER-associated CTRP1 can regulate apoptosis by recruiting DRP1 to the MAM. Finally, mitochondrial fission and fusion defects are known to cause neuronal degeneration, and a previous report showed that Drp1 deletion leads to neuronal degeneration in Pcp2-Cre;Drp $1^{f / f l}$ mice, in which Drp1 is specifically knocked out in $\mathrm{PCs}{ }^{38,39}$. We showed that the Pcp2-Cre;Ctrp $1^{f / f}$ mice also exhibit neuronal degeneration of PCs. Our data thus collectively indicate that ER-associated CTRP1 contributes to neuronal survival and that the mitochondrial fission defects triggered by CTRP1 deficiency contribute to resistance to cell death and neuronal degeneration.

In conclusion, we herein demonstrated that CTRP1 acts as an ER membrane protein to regulate mitochondrial fission via an interaction with DRP1 and identified a potential mechanism wherein the recruitment of DRP1 by CTRP1 could possibly enhance Drp1 ring assembly. We also showed that CTRP1 deficiency induces apoptotic resistance and neuronal degeneration and thereby phenocopies DRP1 ablation. We thus propose a novel role for the cytosolic form of CTRP1. This work provides new insight into the etiology of human disorders associated with mitochondrial fission defects.

\section{REFERENCES}

1. Muendlein, A. et al. The novel adipokine CTRP1 is significantly associated with the incidence of major adverse cardiovascular events. Atherosclerosis 286, 1-6 (2019).

2. Shen, L., Wang, S., Ling, Y. \& Liang, W. Association of C1q/TNF-related protein-1 (CTRP1) serum levels with coronary artery disease. J. Int. Med. Res. 47, 2571-2579 (2019).

3. Shabani, P. et al. Circulating level of ctrp1 in patients with nonalcoholic fatty liver disease (nafld): Is it through insulin resistance? PLoS ONE 10, e0118650 (2015).

4. Janowska, J. D. C1q/TNF-related protein 1, a multifunctional adipokine: an overview of current data. Am. J. Med. Sci. 360, 222-228 (2020).

5. Tang, J. N. et al. Plasma levels of $\mathrm{clq} /$ tnf-related protein 1 and interleukin 6 in patients with acute coronary syndrome or stable angina pectoris. Am. J. Med. Sci. 349, 130-136 (2015).

6. van Hinsbergh, V. W. \& Eringa, E. C. C1q/tnf-related protein 1: a novel link between visceral fat and athero-inflammation. Eur. Heart J. 37, 1772-1774 (2016).

7. Wang, $\mathrm{H}$. et al. Serum levels of $\mathrm{c} 1 \mathrm{q} /$ tnf-related protein-1 (ctrp-1) are closely associated with coronary artery disease. BMC Cardiovasc. Disord. 16, 92 (2016).

8. Yuasa, D. et al. Association of circulating c1q/tnf-related protein 1 levels with coronary artery disease in men. PLoS ONE 9, e99846 (2014).

9. Yang, Y. et al. Association between c1q/tnf-related protein-1 levels in human plasma and epicardial adipose tissues and congestive heart failure. Cell. Physiol. Biochem. 42, 2130-2143 (2017).

10. Kanemura, N. et al. C1q/tnf-related protein 1 prevents neointimal formation after arterial injury. Atherosclerosis 257, 138-145 (2017).

11. Lasser, G. et al. C1qtnf-related protein-1 (ctrp-1): a vascular wall protein that inhibits collagen-induced platelet aggregation by blocking vwf binding to collagen. Blood 107, 423-430 (2006).

12. Liu, Z. H. et al. C1q/tnf-related protein 1 promotes endothelial barrier dysfunction under disturbed flow. Biochem. Biophys. Res. Commun. 490, 580-586 (2017).

13. Yuasa, D. et al. C1q/tnf-related protein-1 functions to protect against acute ischemic injury in the heart. FASEB J. 30, 1065-1075 (2016).

14. Jeon, J. $\mathrm{H}$. et al. A novel adipokine CTRP1 stimulates aldosterone production. FASEB J. 22, 1502-1511 (2008).

15. Sora, $\mathrm{H}$. et al. $\mathrm{C} 1 \mathrm{q} / \mathrm{TNF}$-a-related protein 1 (CTRP1) maintains blood pressure under dehydration conditions. Circ. Res. 123, e5-e19 (2018).

16. Feng, $H$. et al. CTRP3 promotes energy production by inducing mitochondrial ROS and up-expression of PGC-1a in vascular smooth muscle cells. Exp. Cell Res. 341, 177-186 (2016)

17. Zhang, C. L. et al. Globular CTRP3 promotes mitochondrial biogenesis in cardiomyocytes through AMPK/PGC-1a pathway. Biochim. Biophys. Acta 1861, 3085-3094 (2017).

18. Park, S. Y. et al. C1q tumor necrosis factor a-related protein isoform 5 is increased in mitochondrial DNA-depleted myocytes and activates AMP-activated protein kinase. J. Biol. Chem. 284, 27780-27789 (2009).

19. Innamorati, G. et al. GIP, a G-protein-coupled receptor interacting protein. Reg. Pep. 109, 173-179 (2002).

20. Chan, D. C. Mitochondrial dynamics and its involvement in disease. Annu. Rev. Pathol. 15, 235-259 (2020).

21. Zhang, Q. et al. ROCK1 induces dopaminergic nerve cell apoptosis via the activation of Drp1-mediated aberrant mitochondrial fission in Parkinson's disease. Exp. Mol. Med. 51, 1-13 (2019). 
22. Veeresh, P. et al. Endoplasmic reticulum-mitochondria crosstalk: from junction to function across neurological disorders. Ann. N. Y. Acad. Sci. 1457, 41-60 (2019).

23. Cho, H. M. \& Sun, W. Molecular cross talk among the components of the regulatory machinery of mitochondrial structure and quality control. Exp. Mol. Med. 52, 730-737 (2020).

24. Chang, C. R. \& Blackstone, C. Cyclic AMP-dependent protein kinase phosphorylation of Drp1 regulates its GTPase activity and mitochondrial morphology. J. Biol. Chem. 282, 21583-21587 (2007).

25. Otera, H., Miyata, N., Kuga, O. \& Mihara, K. Drp1-dependent mitochondrial fission via MiD49/51 is essential for apoptotic cristae remodeling. J. Cell Biol. 212, 531-544 (2016).

26. Wolf, C. et al. Redox modifications of proteins of the mitochondrial fusion and fission machinery. Cells 9, 815 (2020).

27. Friedman, J. R. et al. ER tubules mark sites of mitochondrial division. Science 334, 358-362 (2011)

28. Korobova, F., Ramabhadran, V. \& Higgs, H. N. An actin-dependent step in mitochondrial fission mediated by the ER-associated formin INF2. Science 339, 464-467 (2013).

29. Manor, U. et al. A mitochondria-anchored isoform of the actin-nucleating spire protein regulates mitochondrial division. elife 4, 1-27 (2015).

30. Duan, C. et al. Drp1 regulates mitochondrial dysfunction and dysregulated metabolism in ischemic injury via Clec16a-, BAX-, and GSH- pathways. Cell Death Dis. 11, 251 (2020).

31. Lim, Y., Dorstyn, L. \& Kumar, S. The p53-caspase-2 axis in the cell cycle and DNA damage response. Exp. Mol. Med. 53, 517-527 (2021).

32. Ma, X., Yu, M., Hao, C. \& Yang, W. Shikonin induces tumor apoptosis in glioma cells via endoplasmic reticulum stress, and Bax/Bak mediated mitochondrial outer membrane permeability. J. Ethnopharmacol. 263, 113059 (2020).

33. Wieckowski, M. R. et al. Isolation of mitochondria-associated membranes and mitochondria from animal tissues and cells. Nat. Protoc. 4, 1582-1590 (2009).

34. Verfaillie, T. et al. PERK is required at the ER-mitochondrial contact sites to convey apoptosis after ROS-based ER stress. Cell Death Differ. 19, 1880-1891 (2012).

35. Viranita, T. et al. The OPA1-dependent mitochondrial cristae remodeling pathway controls atrophic, apoptotic, and ischemic tissue damage. Cell Metab. 6, 834-844 (2015).

36. Yin, X. M. et al. Bid-deficient mice are resistant to Fas-induced hepatocellular apoptosis. Nature 400, 886-891 (1999).

37. Iwata, R., Casimir, P. \& Vanderhaeghen, P. Mitochondrial division ensures the survival of postmitotic neurons by suppressing oxidative damage. Science 369, 858-862 (2020).

38. Ishihara, N. et al. Mitochondrial fission factor Drp1 is essential for embryonic development and synapse formation in mice. Nat. Cell Biol. 8, 958-966 (2009).

39. Kodavati, M., Wang, H. \& Hegde, M. L. Altered mitochondrial dynamics in motor neuron disease: an emerging perspective. Cells 9, 1065 (2020).

40. Ingerman, E. et al. Dnm1 forms spirals that are structurally tailored to fit mitochondria. J. Cell Biol. 170, 1021-1027 (2005).

41. Legesse-Miller, A., Massol, R. H. \& Kirchhausen, T. Constriction and Dnm1p recruitment are distinct processes in mitochondrial fission. Mol. Biol. Cell 14, 1953-1963 (2003).

42. Yedida, G., Milani, M., Cohen, G. M. \& Varadarajan, S. Apogossypol-mediated reorganisation of the endoplasmic reticulum antagonises mitochondrial fission and apoptosis. Cell Death Dis. 10, 521 (2019).

43. Yoon, Y., Pitts, K. R. \& McNiven, M. A. Mammalian dynamin-like protein DLP1 tubulates membranes. Mol. Biol. Cell 12, 2894-2905 (2001).

44. Mears, J. A. et al. Conformational changes in Dnm1 support a contractile mechanism for mitochondrial fission. Nat. Struct. Mol. Biol. 18, 20-26 (2011).

45. Vásquez-Trincado, C. et al. Mitochondrial dynamics, mitophagy and cardiovascular disease. J. Physiol. 594, 509-525 (2016).
46. Kornmann, B. et al. An ER-mitochondria tethering complex revealed by a synthetic biology screen. Science 325, 477-481 (2009).

47. de Brito, O. M. \& Scorrano, L. Mitofusin 2 tethers endoplasmic reticulum to mitochondria. Nature 456, 605-610 (2008)

48. Elgass, K. D. et al. Analysis of ER-mitochondria contacts using correlative fluorescence microscopy and soft X-ray tomography of mammalian cells. J. Cell Sci. 128, 2795-2804 (2015).

49. Arasaki, K. et al. A role for the ancient SNARE syntaxin 17 in regulating mitochondrial division. Dev. Cell 32, 304-317 (2015).

50. Wei, K. J. et al. Receptor-mediated Drp1 oligomerization on endoplasmic reticulum. J. Cell Biol. 216, 4123-4139 (2017).

\section{ACKNOWLEDGEMENTS}

This work was supported by a National Research Foundation of Korea (NRF) grant funded by the Korean government (MSIP) (Nos. 2021M3E5E7023628, 2020R1A3B2079811 and 2018R1D1A1B07050561).

\section{AUTHOR CONTRIBUTIONS}

Conceptualization: S.K.S., Y.Y., and G.T.O.; Methodology, formal analysis, and writing original draft: S.K.S. and G.T.O.; Investigation: S.K.S., S.S., and J.Y.; Writing - review and editing: G.T.O., H.C., D.C.C., K.R., and K.S.L.; Funding acquisition and supervision: G.T.O.

\section{COMPETING INTERESTS}

The authors declare no competing interests.

\section{ADDITIONAL INFORMATION}

Supplementary information The online version contains supplementary material available at https://doi.org/10.1038/s12276-021-00701-z.

Correspondence and requests for materials should be addressed to Young Yang or Goo Taeg Oh.

Reprints and permission information is available at http://www.nature.com/ reprints

Publisher's note Springer Nature remains neutral with regard to jurisdictional claims in published maps and institutional affiliations.

(i) Open Access This article is licensed under a Creative Commons Attribution 4.0 International License, which permits use, sharing, adaptation, distribution and reproduction in any medium or format, as long as you give appropriate credit to the original author(s) and the source, provide a link to the Creative Commons license, and indicate if changes were made. The images or other third party material in this article are included in the article's Creative Commons license, unless indicated otherwise in a credit line to the material. If material is not included in the article's Creative Commons license and your intended use is not permitted by statutory regulation or exceeds the permitted use, you will need to obtain permission directly from the copyright holder. To view a copy of this license, visit http://creativecommons. org/licenses/by/4.0/.

(c) The Author(s) 2021 